\title{
Musique et langage sacrés dans la tradition védique
}

\section{Marius Schneider}

\section{(2) OpenEdition}

\section{Journals}

Édition électronique

URL : http://journals.openedition.org/ethnomusicologie/2435

ISSN : 2235-7688

\section{Éditeur}

ADEM - Ateliers d'ethnomusicologie

Édition imprimée

Date de publication : 1 janvier 1992

Pagination : 151-182

ISBN : 978-2-8257-0456-1

ISSN : $1662-372 X$

\section{Référence électronique}

Marius Schneider, «Musique et langage sacrés dans la tradition védique », Cahiers d'ethnomusicologie [En ligne], 5 | 1992, mis en ligne le 15 décembre 2011, consulté le 19 avril 2019. URL : http:// journals.openedition.org/ethnomusicologie/2435 


\title{
MUSIQUE ET LANGAGE SACRÉS DANS LA TRADITION VÉDIQUE
}

\author{
Marius Schneider
}

Né à Haguenau en Alsace, Marius Schneider (1903-1982) étudia la philologie, la musicologie, le piano et la composition à Strasbourg (1921-1924), à Paris (19241927) et à Berlin (1927-1930) ${ }^{1}$. C'est dans cette dernière ville qu'il acheva son cursus universitaire par une dissertation sur L'Ars nova en Italie et en France (1930). Cette étude est la première d'une série de recherches sur la polyphonie, qu'il allait ensuite élargir aux expressions musicales extra-européennes. La documentation qu'il accumula à cet effet constitua un fonds important des Archives phonographiques de Berlin, dont il devint le directeur en 1932, ainsi que la base de son travail de synthèse Geschichte der Mehrstimmigkeit (1934-35, 1969), fortement marqué par les courants de pensée comparatiste et diffusionniste alors prépondérants en Allemagne. Au sortir de la guerre, il est nommé à la tête de la section dédiée aux musiques traditionnelles et populaires de l'Instituto Español de Musicologia à Barcelone. C'est de cette période que datent certains de ses essais les plus marquants, sinon toujours les plus intelligibles, parmi lesquels il faut citer $\mathrm{El}$ origen musical de los animales - simbolos en la mitologia y la escultura antiguas (1946), La danza de espadas y la tarantela (1948) et le fameux Singende Steine (1955), dont la traduction française a été publiée en 1976 sous le titre Le chant des pierres ${ }^{2}$.

Comparatiste, Schneider le restera toute sa vie, et sa vaste bibliographie témoigne de sa connaissance des cultures musicales les plus diverses: méditerranéennes (Italie, Espagne, monde arabo-andalou et berbère), négro-africaines (Ouganda, Caméroun, Togo, Ghana), latino-américaines (Brésil, Argentine), asiatiques (Caucase, Iran, Inde, Chine, Philippines), jusqu'à la musique des Aborigènes australiens, à laquelle il consacra un article. Mais cette ample documentation est avant tout pour lui la matière première de recherches totalement originales sur des thèmes tels que l'origine des traditions populaires, les anciennes cosmogonies, le symbolisme sonore et la signification de la musique de façon générale. Titulaire de la chaire de musicologie à l'Université de Cologne de 1954 à 1970, il publie alors un

Cette préface emprunte certaines données biographiques à l'introduction d'Elémire Zolla au recueil de traductions italiennes d'articles de Schneider paru sous le titre Il Significato della Musica (Milano: Rusconi, 1970).

2 La bibliographie la plus complète de l'œuvre de Schneider est celle publiée en 1969 par Robert Günther: «Special bibliography: Marius Schneider», Ethnomusicology, vol. 13 (3): 518-26. Elle ne fait cependant état que de ses publications parues jusqu'en 1968. 
nombre impressionnant d'études, dont les plus connues demeurent ses contributions à plusieurs dictionnaires et encyclopédies: Die Musik in Geschichte und Gegenwart de Friedrich Blume (1949-68), The New Oxford History of Music (1954), l'Encyclopédie de la musique de F. Michel, F. Lesure et V. Fédorov (195861), le volume I de l'Histoire de la musique de l'Encyclopédie de la Pléiade (1960) et l'Encyclopédie des musiques sacrées de Jacques Porte (1968) notamment.

Ses affinités avec l'Orient, son intérêt pour le monde des mythes et, plus encore, son goût pour les spéculations métaphysiques allaient inévitablement le rapprocher de la tradition hindoue, et ses derniers écrits font état de nombreuses références aux textes fondamentaux de l'hindouisme que sont les Veda et les Upanishad. C'est à cette catégorie qu'appartient l'article que nous publions ci-dessous. Il s'agit du texte inédit d'une conférence donnée par Schneider le 31 août et le $1^{\mathrm{er}}$ septembre 1971 à l'Instituto Ticinese di Alti Studi de Lugano dans le cadre d'un colloque organisé par le professeur Elémire Zolla sur le thème des «Langues et musiques sacrées ». Il n'est pas sûr que l'auteur eut souhaité publier ce texte sous cette forme, et de nombreux passages sont demeurés obscurs aux différents spécialistes auxquels nous l'avons soumis, notamment en l'absence de références précises. Il constitue néanmoins un chapitre important de l'œuvre de Marius Schneider, une æuvre qui apparaît aujourd'hui comme une des plus étonnantes et des plus audacieuses de l'histoire de l'ethnomusicologie.

Laurent Aubert

\section{Le son créateur}

Le verbe a créé le monde; mais il le maintient aussi. Donc, pour comprendre la nature et la position cosmogonique du chant et du langage rituels de la tradition védique, il est indispensable de les analyser parallèlement à la création, à l'évolution et à l'organisation du monde.

Alors que la tradition judéo-chrétienne nous présente un créateur qui initie son œuvre avec l'éclosion d'une première lumière éclairant les ténèbres, ou avec la parole qui est Dieu et en Dieu, la genèse védique parle d'un son indéfinissable. Ce son est le premier souffle, et en dernier lieu le soleil primordial qui chante. Ce son solaire est censé être immortel et inaudible. Il est le premier brahman, placé en dehors du temps et de l'espace, tonnerre lumineux constituant la Vérité absolue.

Les débuts de la création reposent donc sur une contradictio in se: un son qui ne sonne pas. Ce brahman est - humainement parlant - l'activité qui se place entre la naissance d'une idée et la formulation audible de cette idée. C'est une sorte de son intérieur, le son de la pensée.

Au cours de la création, il est suivi d'un deuxième brahman, constitué par un son perceptible. Le premier brahman est privé de forme. Il réside dans le premier élément, appelé $\bar{a} k \bar{a} c ̧ a$, dont la seule qualité consiste à être porteur d'un son inaudible. Le deuxième brahman se réalise dans l'élément air, qui rend son audible. Mais par ce fait, ce deuxième brahman prend, par opposition au premier, 
une forme, et ceci l'éloigne de la Vérité absolue, car selon la philosophie du Vedanta, la Vérité absolue n'a pas de forme, car tout ce qui épouse une forme appartient au monde de la $m \bar{a} y \bar{a}$, à l'illusion des sens, à la représentation que l'homme se fait du monde. Cependant, étant donné la nature acoustique du deuxième brahman, cette première formule exprime la Vérité de la meilleure façon possible, parce qu'elle est quand même encore abstraite. Son matériel est le souffle, sa forme très fuyante et - comme un pur rythme sonore et musical elle est exempte de toute limitation ou spécification linguistique ou visuelle. Ce brahman a un sens, un sens musical, rythmique, bien qu'au point de vue linguistique il soit privé d'une signification déterminée.

Le brahman classique est la syllabe AUM ou OM, dans laquelle le souffle est expiré à partir de $\mathrm{A}$, passant par toute la série des voyelles pour aboutir à $\mathrm{M}$, qui à son tour s'évanouit dans les consonnes S-H. La Chāndogya Upanishad dit: «AUM a tout créé. Elle est la syllabe du consentement» (AMEN).

Cette syllabe sacrée est issue du désir du créateur, c'est-à-dire du son encore inaudible qui, en devenant perceptible, constitue le triangle des trois sons A.U.M. Dans cette trinité existe le créateur et, à côté ou en lui son fils, c'est-àdire la voix du créateur qui exprime dans un saint esprit le désir de créer le monde.

La tradition dit que le premier brahman fit naître dans un lotus le deuxième brahman, qui est le dieu Brahmā. Et celui-ci réfléchissait: «Quelle est la parole par laquelle on peut obtenir l'accomplissement de tous les vœux, afin que tout prenne forme et conscience?» Il pratiqua l'austérité par ascétisme et c'est par un sacrifice que le dieu Brahmā prit naissance et conscience et créa les premières formes. Ces formes constituent la nature du monde primordial: un monde purement acoustique et sans aucune lumière, dans lequel l'extériorisation, je veux dire la formulation audible de tout vœu, de tout souhait est déjà la réalisation. Dans cette première époque de la création, la parole est la première action, et en même temps le premier objet existant, puisque ces objets n'étaient que de purs rythmes sonores. Donc tout-à-fait au début, il n'y avait que le Non-Existant, et de ce Non-Existant, qui devenait une intention ou un souhait, sortaient les premiers rythmes appelés $r s ̦ i-$ les premiers poètes.

Dans la littérature moderne, ces rythmes sont distingués par le terme «nom» (le nom des objets est la nature des objets). La philosophie des Upanishads distingue plus exactement nāma et rūpa, c'est-à-dire nom et forme, ce qui signifie en dernière analyse: son et rythme. Mais ces noms ou ces sons ne sont pas les noms avec lesquels notre langue distingue des objets existants. Ce sont la substance acoustique, pour ainsi dire les mélodies constituant les objets. Sortant de l'élément $\bar{a} k \bar{a} c ̧ a$, la Vérité absolue, ces idées deviennent des rythmes et des formes audibles en entrant dans l'élément air. Alors les idées, d'abord pensées, sont prononcées et deviennent sonores.

Il s'ensuit une conception du créateur, dont la nature est un souffle, le vent, un tourbillon, une parole, un chant ou une bouche, prononçant le monde. Cette bouche passe pour être plus ancienne que la tête et le corps.

La voix d'Eulil (Babylone) est une tempête. Thot (Egypte) pousse $7 \times 10$ cris. Prajāpati, le dieu créateur des brahman fait entendre dix-sept tambours. Sa co- 
lonne vertébrale est formée de rythmes; ses membres et son tronc sont des chants de louange; ses cheveux sonnent le chant du Säma-Veda. Il est évident que ces images ne sont que des moyens littéraires pour exprimer une situation acoustique.

Parfois les textes sont moins imagés, surtout quand il s'agit de montrer le procédé de la création. Prajāpati prononçant la formule AKAHO créa avec A la terre, avec $\mathrm{HO}$ le ciel et avec KA le monde intermédiaire (l'atmosphère). Cette force créatrice lui parvint grâce à une vie ascétique, qui est simplement le silence dans lequel il réussit à accumuler ses forces acoustiques.

Le caractère de ces sons est décrit de manières très différentes. Généralement on l'appelle un chant de louange. "Au début il n'y avait rien, car le monde était enveloppé par la mort. Or la mort est la faim (ou la soif) (pensée antithétique!). C'est alors que la mort (la vache Prajāpati) désira prendre corps. Pour obtenir ce corps, elle créa la volonté d'exister en élevant sa voix par un chant de louange. Ce chant lui inspirait la joie et cette joie produisit les eaux, ou le lait (liquide). La crème de ce lait fut barattée pour en faire la terre. Ceci faisant, le chanteur s'échauffa à tel point que le feu sortit de sa sueur» (Brahmana Upanishad).

Voici une présentation imagée de la genèse des éléments air, eau, terre, feu, produits par une série de rythmes; car ces premiers éléments ne sont pas encore des éléments concrets, mais leur préfiguration sonore.

D'autres traditions décrivent le chant initial comme un cri de nouveau-né, étant donné que le créateur inhérent à l'Anthropocosme est lui-même (au commencement) un nourrisson. Souvent cette voix créatrice est le premier tonnerre ou pluie sonore. Le $R g$-veda parle d'un grand son double, initiant de dualisme universel. Ce dualisme est bien manifeste dans Prajāpati. Ce mort qui chante est une figure dualiste et en même temps la formule antithétique fondamentale: la mort produit la vie, et la vie produit la mort.

De cette pensée antithétique résultent des formules constitutives de la genèse. Ainsi par exemple la «respiration sans souffle», semblable au «son qui ne sonne pas», et qui symbolisent probablement la pure pensée. Au dire du Rg-veda, les débuts ignoraient à la fois l'Être et le Non-Être, la mortalité et l'immortalité. Puis il se forme le brahman «non-né», qui existe sans avoir jamais eu une naissance (puisqu'il est en dehors du temps et de l'espace), mais qui est animé du désir de créer. C'est par l'amour pour un objet encore inexistant que le brahman a créé le temps et l'espace! Tout cela, dit le $R g$-veda, «était une marée ou un flux inconnaissable» dans lequel le brahman «respirait sans souffle dans un grand vide». Ce vide est le premier corps de résonance (la caverne de la mythologie) dont une force, altérée ou créée par le vacuum, s'élevait comme un chant.

Cette situation antithétique et dualiste est le point de départ dans lequel les éléments, qui dans notre logique et dans notre monde concret s'excluent mutuellement, formaient encore un flux rythmique continu, dont la première concrétisation est la chaude humidité, source de toute croissance. L'opposition du feu et de l'eau ne se développe que bien plus tard. Au début règne la formule: le feu dans les eaux, ou le rythme dans le son.

Aussi les dieux créateurs sont-ils souvent symbolisés par un rythme de tambour, ou simplement comme des tambours résonant au milieu des ténèbres du 
monde primordial. Ces dieux jouent du tambour ou sont eux-mêmes des tambours, parce que dans ce premier monde le sujet et l'objet ne sont pas encore différenciés. Le sujet produit l'objet. La voix est l'activité créatrice du sujet, et le son de la voix du sujet est l'hypostase de cette voix, c'est-à-dire son objet. La meilleure formule exprimant ce dualisme est constituée par les trois premiers sons de la série harmonique: base, octave et quinte.

Toutes ces idées restent évidemment abstruses quand on ne les conçoit pas dans le cadre de l'Anthropocosme, car tout l'univers est considéré comme un analogue de l'homme. Cela est essentiel pour la théorie de la connaissance car l'homme est le seul objet qui intérieurement peut être connu et senti par l'intelligence humaine. Les autres objets ne peuvent être connus que d'une manière formaliste, car nous ne savons rien de leur vie intime.

Il reste encore une observation à faire à propos du dualisme. Le dualisme luimême est double, c'est-à-dire qu'à côté du dualisme rythme et son il y en a un autre qui est image et lumière. Quand on parle du rta constant qui est une partie du monde primordial, on mentionne une masse lumineuse, qui rayonne invisiblement dans le fond, ou dans la fontaine, du rta.

Les deux formes du dualisme réunies créeront le son-lumière, qui se manifestera seulement à partir de la première aurore ou du printemps de la création. Pour le moment, nous traitons seulement le début, le minuit ou l'hiver de la création, issu du Nord - astronomiquement parlant - du Cercle polaire du Septentrion. Dans cette situation, leur meilleure formulation possible est acoustique, puisqu'elle reste le plus proche possible de la nature du son créateur. Telle est la forme la plus vraie; car, tout en ayant pris une forme (ce qui est déjà en dehors de la Vérité absolue), elle est une première hypostase du son créateur. Or cette première forme est nommée la tête du sacrifice. Elle est le premier sacrifice, dont le résultat est la création du monde. Cette idée est capitale. Elle domine toute la philosophie et tout le rituel védique. Tout ce que les dieux font, ils le font par le sacrifice; et la substance du sacrifice est le chant. Même les dieux durent accomplir le sacrifice pour gagner l'immortalité! Par un sacrifice ininterrompu, le monde est créé et maintenu en vie.

Le sacrifice est donc en premier lieu l'offrande sonore du souffle vital et en dernier lieu l'expression définitive de la vie dans la mort. Un rite est sans efficacité s'il n'est pas porté par cette parole sacrificielle. Quand on présente une offrande à Agni, il faut que le prêtre dise au moins: "AUM! Ceci pour vous, Agni!». Le sens de cette syllabe AUM est le consentement, un «oui» sans réserve exprimant la renonciation à la Non-Vérité.

\section{Le système cosmogonique}

Le meilleur moyen pour reconstruire le système cosmogonique est une orientation selon les nombres dispersés dans les textes. Les nombres fondamentaux vont de 1 à 10 et, en dernière analyse, correspondent aux dix premiers sons de la 
Fig. 1

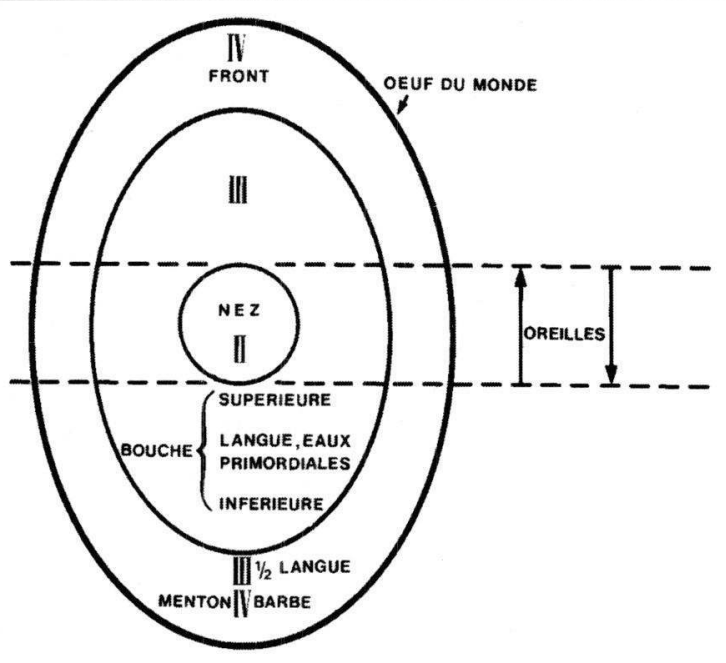

série harmonique. Nous faisons abstraction des nombres supérieurs à dix pour éviter d'embrouiller ce rapide exposé. Selon ce plan, l'univers se développe en dix cercles ou cycles concentriques. Le début est formé par le centre commun à tous les cercles. Le monde primordial s'étend de 0 à $\mathrm{III}^{1} \frac{1}{2}$. Il est en dehors du temps et de l'espace. Avec le cycle IV se forment le temps et l'espace pendant l'«hiver» ou la «minuit» de la création. C'est le premier solstice d'hiver, l'enfance de l'Anthropocosme.

Dans le monde primordial (voir fig. 1), le cycle II, le nez, le souffle, la respiration, la consonance de l'octave constituent le siège du dualisme sonore. Le cycle III renferme le ciel et la terre purement acoustiques formant la bouche de ce monde. Le palais correspond au ciel, la partie inférieure de la bouche à la terre, et entre les deux résonne le tourbillon de la mer primordiale. Dans ces eaux se trouvent toute la création et toutes les fonctions futures qui au cycle III $1 / 2$ seront prononcées par la langue. Le cycle III est la bouche du premier sacrifice. Dans cette image du monde sonore, le dualisme cosmique est représenté par le fait que tous les éléments apparaissent en deux formes: l'une en position «normale» et l'autre renversée. L'ensemble de cette tête primordiale est enfermé dans l'œuf du monde. Les cycles I, II, III et III $1 \frac{1}{2}$ correspondent à la situation fondamentale de la syllabe AUM, composée de trois temps plus un demi temps pour l'expiration.

Dès que la tête primordiale ouvre la bouche et part crever l'œuf du monde, l'Anthropocosme prend naissance comme une création spatiale et temporelle. Il va en grandissant depuis le cycle IV jusqu'à ce que sa tête atteigne le cercle $\mathrm{X}$; en même temps, ses fonctions physiques et psychiques, préexistant dans le rythme sonore de l'œuf, se transforment en matière et organes concrets. C'est ainsi que s'initie la matérialisation progressive de l'univers, qui atteint son point culminant au cycle X, c'est-à-dire pendant l'été de la création (solstice d'été), avant de 


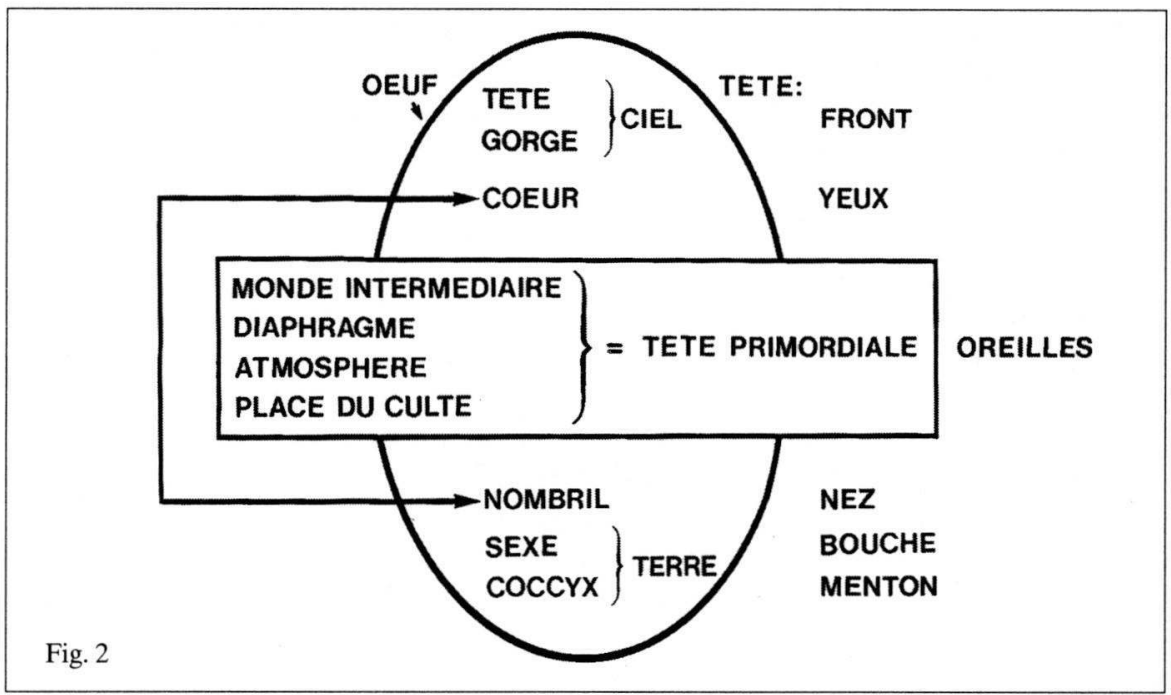

retomber au cycle IV. Entre temps, des parties du monde acoustique deviennent liquides, une partie du liquide se transforme en cristaux à matière cornée et finalement en pierre. Néanmoins, le centre sonore commun à tous les cycles survit dans la voix de la nature. Il y a cependant déjà dans le cycle IV une pétrification prématurée et nécessaire, qui peut être définitive ou passagère. Ceci tient au caractère ambivalent de ce cercle. Il contient une bifurcation, c'est-àdire la possibilité de se raidir définitivement ou passagèrement contre l'omniprésence du son primordial, qui est le sacrifice. Il est le stade préparatoire du cycle VII, qui est l'aurore de la création, conçue comme la libération des forces vitales par le sacrifice.

Pour éviter tout malentendu, j'insiste sur le fait que la tête primordiale enfermée dans l'œuf du monde n'est pas identique à la tête de l'Anthropocosme réalisée dans le temps et l'espace. La tête de l'Anthropocosme est un produit de la tête primordiale. L'Anthropocosme est un phénomène de la $m \bar{a} y \bar{a}$, c'est-à-dire du monde de la Non-Vérité, qui fait naître un ciel et une terre au-dessus et audessous de l'ancien ciel et de la terre acoustiques constituant toujours le centre et l'embryon de l'univers. Le nouveau ciel et la nouvelle terre sont formés par les deux parties de l'œuf crevé (voir figure 2)!

Et tout cet ensemble forme encore une tête où le front est le ciel, la bouche la terre, les yeux le soleil et la lune, c'est-à-dire le monde intermédiaire, la place du culte, encadré par les oreilles, qui perçoivent les sons du monde primordial.

L'Anthropocosme est l'œuvre de la $m \bar{a} y \bar{a}$ qui nous cache la tête primordiale, c'est-à-dire l'embryon universel; mais elle ne le détruit pas. Et par le fait que cet embryon reste toujours au centre, commun à tous les cycles de l'univers, le monde acoustique créateur est toujours présent. Il est situé au plexus solaire de l'Anthropocosme. Cette présence indestructible des origines et du premier 
temps passé ne se manifeste pas seulement dans les sons du cœur de l'embryon, mais aussi dans l'omniprésence des principes fondamentaux dans l'organisation du monde concret, le monisme, le dualisme et la trichtomie.

Notons encore un autre point fort important. Entre les cycles III et IV se placent les $3 \frac{1}{2}$ morae. Ce sont trois étapes de la pensée correspondant aux trois lettres de la syllabe A-U-M plus le temps d'expiration. Ces étapes figurent dans notre système comme cycle III $1 / 2$. Mais les Upanishad disent que la durée de ces 3 temps et demi est de 7 mätră. C'est pourquoi, dans le monde existant dans le temps et l'espace, le 31/2 vaut 7 temps, rythmiquement. Nous désigneront ce 7 procédant du cycle III $1 \frac{1}{2}$ comme le 7 intérieur, afin de le distinguer du cycle VII. Le III $1 \frac{1}{2}$ correspond au début de l'hiver, le VII au printemps.

Astronomiquement, le groupe du 7 intérieur ou primordial renferme entre autres la Grande Ourse du cercle polaire et 7 rythmes originaux, que l'on appelle parfois $R s c i$, les premiers poètes ou mètres de poésie. Le III $12 / 2$ ou 7 intérieur est la position originelle de la langue qui émet les sons S, H ou Ș. C'est le moment dans lequel la Non-Vérité (le dualisme) commence à voiler la Vérité pour faire surgir notre monde concret. Cette duplicité de la Vérité et de la NonVérité est symbolisée par deux serpents (ou un double serpent) qui, s'entrecroisant, montent et descendent le long d'un bâton (parfois invisible). Le bâton est la Vérité, les deux serpents la Non-Vérité qui tourne autour de la Vérité (voir fig. 3).

Voici les critères essentiels des dix cycles:

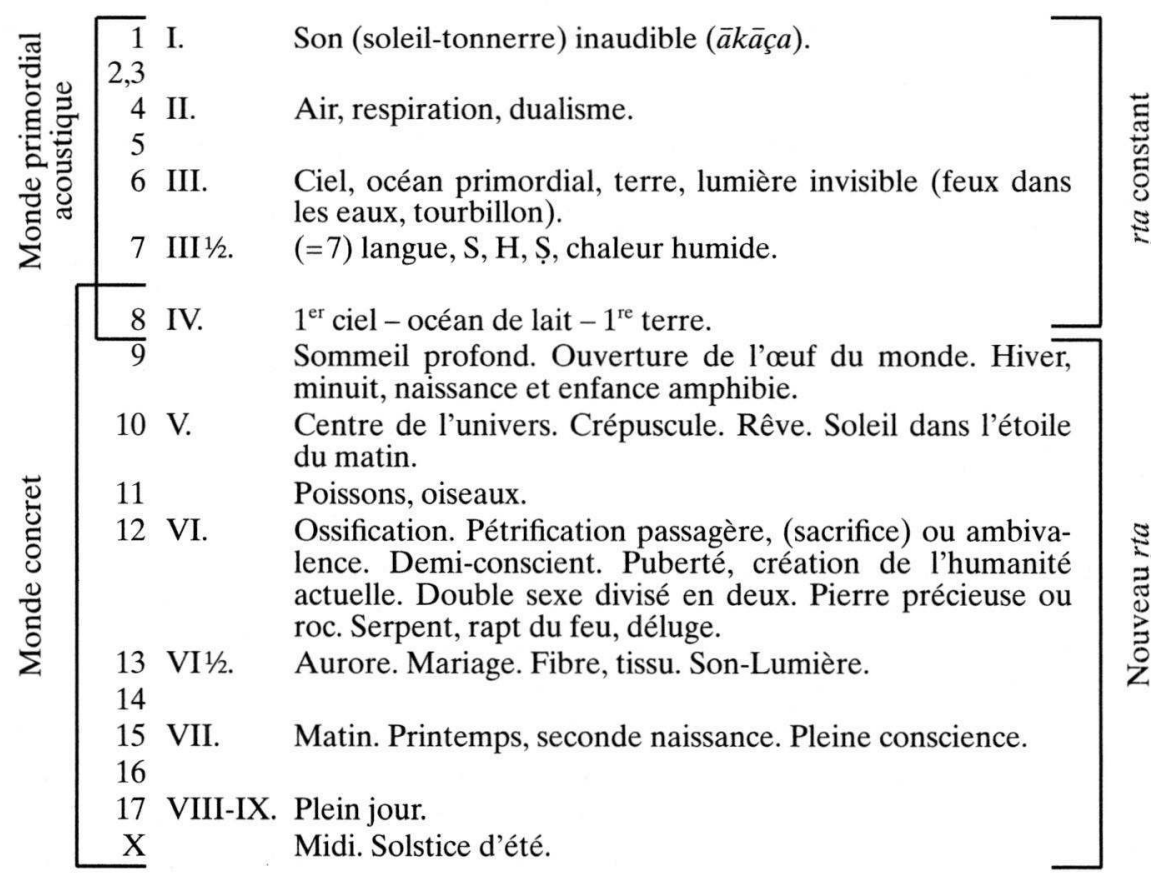




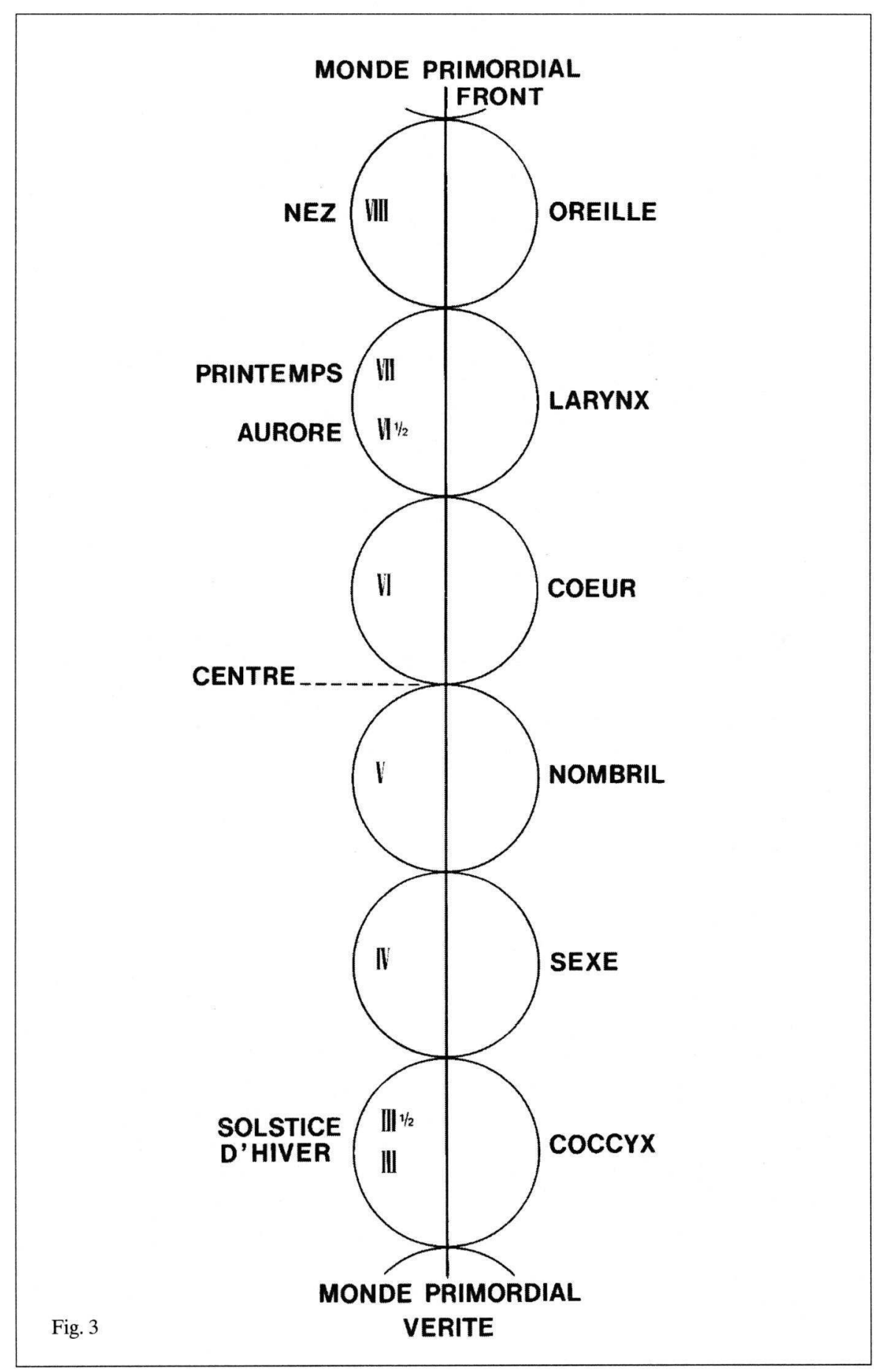


Mais comme l'univers se développe en cercles concentriques, la disposition réelle est:

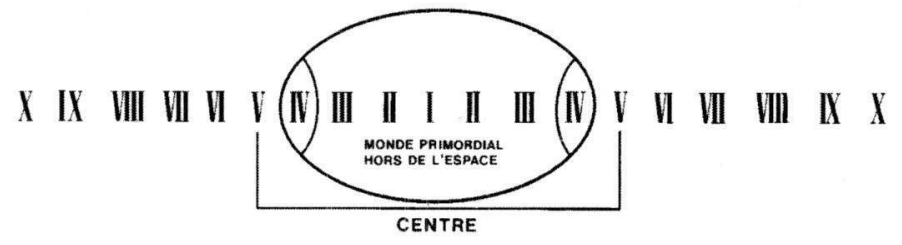

Fig. 4

Les relations entre le monde primordial et le monde concret sont donc

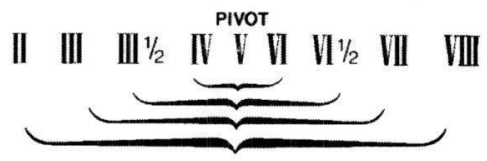

Fig. 5

A chacun de ces cycles correspond une partie du jour et de l'année, un âge, un état de conscience, un paysage, une sonorité et des rites caractéristiques. Au IV commence la matérialisation du monde. Au VI $1 \frac{1}{2}$ se place l'aurore censée être un son-lumière. Jusqu'au cycle VI, qui inclut le Paradis biblique, la conscience de l'homme est une partie intégrale de l'Anthropocosme. Son expérience est complète. A partir de VI $1 \frac{1}{2}$, il commence - intellectuellement - à se séparer de cette intégration et à s'assujettir la nature et les animaux, mais sa conscience intellectuelle devient incomplète (hors de la nature).

\section{Les eaux}

Bien que le monde primordial soit purement acoustique, la description que l'on en donne se fait essentiellement par des images concrètes. Il est évident que la notion des eaux primordiales, du ciel et de la terre primordiaux, ainsi que des nuages, de la pluie, du lait, et même de la première pierre ou de la caverne de résonance du monde primordial, sont simplement des moyens techniques pour exprimer un état de choses qui littérairement ne peut être exprimé autrement. En fait, tous ces éléments sont des images symbolisant des fonctions, c'est-à-dire les rythmes originels purement acoustiques.

Ceci change à partir de l'apparition de la première terre semi-concrète au quatrième cycle. Dans cette période de la création, les eaux représentent tantôt le murmure des eaux, tantôt les véritables eaux, mais dont la voix laisse deviner la présence créatrice du son (syllabe AUM) qui les rend fécondes. Aussi l'eau 
passe pour être la première matérialisation du son créateur. Le $R g$-veda dit que la Sainte Parole est habillée d'eau, ou que les eaux sont pénétrées du son mystique. Les Dogon de l'Afrique occidentale parlent d'un chant créateur humide, ou plein d'eau.

C'est Prajāpati, le dieu créateur védique, qui, pratiquant des austérités (sacrifices), créa au cycle III la triple science, et avec la voix de cette triple science il pénétra les eaux. Ensuite il désira sa multiplication, et voici que l'œuf du monde se forma sur la surface de l'océan. Souvent, la notion de «pleuvoir» équivaut à «créer». L'eau, issue et pénétrée de la syllabe AUM, est la seconde parole créatrice. L'eau est censée être le séjour des morts qui créent la vie. Dans les rites, le chant purifie le souffle et l'âme, alors que l'eau purifie le corps. Dans la bouche, les deux éléments (son et eau) se réunissent lorsque la langue est, selon le $R g$-veda, habillée d'eau.

Si le rythme sonore constitue la meilleure formulation possible de la Vérité, l'eau possède également cette faculté, mais à un moindre degré. Tout en ayant une tendance naturelle vers la forme sphérique ou tortueuse, elle renonce à une forme propre. Elle s'accommode de formes préexistantes, et dans la solitude son bruit dirige l'homme vers son créateur. Elle n'est pas soumise à la contrainte des formes du monde de la Non-Vérité. De même que deux voix musicales peuvent résonner simultanément sans s'effacer mutuellement, deux courants d'eau peuvent se traverser ou se frotter sans paroi de séparation (Gulf Stream) sans se mêler.

Le philosophe chinois Laozi glorifie l'eau pour sa modestie. Elle se rend toujours à la place la plus basse. Elle est le symbole de la tranquilité d'âme. Son murmure fournit les meilleures conditions pour acquérir des connaissances supérieures par méditation. Elles est proche du Tao. Les musiciens accourent aux fontaines pour se faire inspirer les thèmes de leurs compositions.

Ce que les Dogon appellent la «parole pleine d'eau» se retrouve dans le $R g$ $v e d a$ sous le nom du flux inconnaissable des eaux. Ces eaux sont $v \bar{a} c$, la voix sonore qui est considérée comme la première Née dans les ondes sonores de l'océan primordial, c'est-à-dire dans la bouche de la tête symbolisant le monde acoustique. L'Atharva-veda nous dit que cette $v \bar{a} c$ parlait d'abord un langage absolument incompréhensible. Cela veut dire qu'au début, la langue ignorait et les mots dotés d'une signification précise et les formes grammaticales. Elle parlait des rythmes purement cosmiques. Des rythmes de ce gnere s'employaient en effet dans les rites, où les chanteurs imitaient le bruit de l'eau, du tonnerre, de la pluie, voire des animaux.

Reproduisant le timbre et le rythme de ces voix, on s'identifiait avec l'essence acoustique des forces cosmiques. Il va de soi que cette imitation ne peut se borner à un simple procédé technique, puisqu'elle doit aboutir à une identification du chanteur avec les sons de l'Anthropocosme. Le son est le point de rencontre; c'est par lui que se réalise la communion de tous les êtres. Une tel son n'est pas un simple appel à une divinité, mais un essai de s'identifier à elle. Et ceci grâce à la nature microcosmique de la voix humaine, car en dehors de la voix, la faculté d'imitation de l'homme est bien plus limitée. Aussi dans les rites, les travestissements du corps ont-ils bien moins d'importance que les différents timbres de la voix. 
La reproduction juste du timbre et du rythme, ce que l'on appelle la «voix juste», est la base de la connaissance intérieure des choses. Il serait cependant erroné de considérer ce procédé comme une sorte d'agnosticisme musical. Et ceci pour deux raisons:

1. Les rythmes sonores du monde primordial nocturne, privé d'expression visuelle, ne peuvent être compris que comme des prototypes du mouvement.

2. Le mouvement sonore, lui aussi, a sa logique qui reste autonome, même quand il est accompagné d'un texte (se déroule dans un temps déterminé, fonctions tonales, séquences, accelerando, rallentando, etc.). C'est pourquoi le langage rituel, même quand il est composé de phrases littéraires claires et distinctes, doit toujours être porté par un flux musical élémentaire et naturel.

Par ce flux élémentaire et naturel, j'entends quatre sortes d'émissions sonores, qui se produisent:

a. durant la reproduction exacte d'un bruit de la nature.

b. L'expression vocale spontanée de l'homme comme réaction immédaite sur une impression physique ou psychique (joie, douleur, terreur, etc.).

c. Le transport de rythmes visuels et inaudibles sur le plan acoustique (mouvement ondulant, vagues, rivages).

d. L'action de battre un tambour ou de souffler dans un instrument, à condition que cet instrument soit construit à base d'un animal de sacrifice (peau), car toutes les émissions sonores rituelles sont des sacrifices.

Toutes ces sonorités ont donc un sens, bien qu'elles n'aient pas une signification déterminée, tel un mot du langage. Ce sont des forces beaucoup moins limitées qaue les mots emprisonnés dans une détermination linguistique. Elles sont $v \bar{a} c$ (vox) parlant un langage logiquement incompréhensible, mais bien compréhensible par son ton, à tel point qu'il est intelligible et par les hommes et par les animaux.

Le support de ce mouvement sonore est l'air. Sa racine primordiale se trouve dans le tourbillon de l'océan du cycle III. L'eau, mise en branle par le vent, avale l'air et forme un entonnoir sonore ou, selon une autre image, elle creuse un puits, la fontaine du rta, dont la base se lève et se baisse périodiquement et avec plus ou moins de violence. Cette base est le diaphragme du monde. Cette partie du monde primordial est le point de départ du langage en syllabes mystiques, dont l'exécution rituelle a eu lieu dans le cinquième cycle, mais qui pratiquement représente dans le monde concret l'ensemble du monde primordial. Ici la syllabe AUM apparaît prononcée sur le ton caractéristique de ce cycle. On l'appelle «la syllabe blanche», émise en expirant, et servant en même temps à la purification. Par contre, la syllabe HUM, appelée «bleue», est émise en inspirant l'air. Ceci faisant, l'homme avale le $\mathrm{H}$, le son spirituel par excellence. Cette syllabe n'a pas de valeur sémantique, mais son sens est: "Il est, Il existe», c'est-à-dire le brahman existe, car quand Brahmā retire le souffle, il avale et détruit le monde de la $m \bar{a} y \bar{a}$, c'est-à-dire de la Non-Vérité, et lorsque le souffle est retenu, il se produit un vide à l'intérieur de l'homme, et c'est là où l'on perçoit l'immuable Vérité. Ce moment culminant est la syllabe rouge AH qui, supprimant toute pensée intellectuelle, permet le contact avec le monde supra-intellectuel et supra-sensible. 
C'est ce qu'on appelle la «respiration intermédiaire», ou l'essieu (l'axe) cosmique. Ceci faisant, le yogi arrondit la poitrine, formant la caisse de résonance traversée par la sușumna (l'axe du monde et de la Vérité). La respiration est arrêtée et les sons intérieurs deviennent audibles. En ce moment, on voit le «corps sonore de la Vérité» sans qu'uen pensée intellectuelle puisse entraver l'indétermination de ces sons. La valeur ou la direction de l'action d'une syllabe dépend du ton comme expression psychologique de la situation donnée.

\section{Le lait et la solidification de la matière}

Le langage des syllabes mystiques pratiqué dans le cycle $\mathrm{V}$ aspire à réaliser les sons avec le plus grand réalisme. Dans le sixième cycle du monde concret, cette formulation subit une notable transformation. L'émission des syllabes devient plus mélodique, le flux plus contenu, les syllabes sont souvent liées les unes aux autres et les imitations des syllabes naturelles sont plus stylisées. Les contrefactures prennent un accent plus spécifiquement humain. Le sixième cycle est caractérisé par une sorte de totémisme en déclin, où l'homme se différencie d'une manière de plus en plus nette de l'animal pour en devenir le maître. Il parle encore aux animaux une sorte de langage conforme aux bêtes, mais avec un accent humain. Ceci faisant, l'homme se dégage de son implication à la nature. C'est le cycle de la culture de la terre et de l'élevage.

Le langage du cycle VI tire son origine du cycle IV. Lorsque la bouche de la tête primordiale s'ouvrit, l'œuf du monde creva, l'océan intérieur se déversa et engendra l'océan de lait, c'est-à-dire la première forme de la voie lactée. Le ciel et la terre primordiaux, acoustiques, provoquaient un ciel et une terre semi-concrets, qui dorénavant s'éloigneront dans la mesure où l'Anthropocosme s'élargit. Entre le nouveau ciel et la nouvelle terre du cycle IV se développait un monde intermédiaire, la première Atmosphère constituée par les cycles III et III 1/2.

Cet océan intermédiaire rempli de lait ou d'eau douce fut baratté au moyen du moulinet (le futur poteau de sacrifice ou axe du monde), avec lequel les dieux produisirent le tourbillon résonant du cycle III. C'est ainsi que, par le son et le mouvement de la première matière encore toute liquide, se formaient le premier ciel et la première terre semi-concrets. La force créatrice qui fit naître le cycle IV est le ton sur lequel sonnait la syllabe AUM. Ce ton avait le timbre de la voix d'une vache, dont on dit qu'elle a donné le lait nécessaire à la création du cycle IV. Aussi ce cycle est-il la position cosmogonique par excellence de la syllabe AUM. Elle se trouve sur la frontière entre la Vérité et la Non-Vérité; elle est, dans notre monde concret, la seule formulation possible de la Vérité. C'est pourquoi le $R g$-veda dit: «La vache laitière est la Vérité». C'est devant le poteau du sacrifice (axe du monde) que le chant des rși a produit cette vache. Du souffle sonore de cette vache sortent les eaux, l'éclair et le tonnerre. Son pis est le dieu de la pluie féconde; ses tettes sont les éclairs. C'est Brahmā qui l'a fait sortir du ventre de Varuna, l'océan primordial (ventre = bouche, III, devient centre). 
D'autres versions disent que son pis est le nuage, et ses tettes rathāntara et brhat (formes du säman). C'est de ces tettes, dit l'Atharva-veda, que les dieux ont trait le monde.

Le même Atharva-veda attribue à la vache AUM le cycle I qui est également le nombre sacré du tonnerre inaudible. Mais, de même que le tonnerre, la vache obéit au nombre 4 , qui est le premier nombre du monde créé ( $4^{\mathrm{e}}$ cycle). Sa place correspond aux quatre fleuves ou aux quatre trous par lesquels les eaux sonores du rta se déversent sur la terre et dans l'océan.

Mais tout cela ne forme que le plan arrière du cycle IV, où ce lait, le miel et le chant, qui lui correspondent, est réalisé. Le paysage du sixième cycle est le Paradis, y compris son ambivalence qui, sur le plan du Paradis, apparaît comme la tentation par le serpent. Ce serpent est le serpent ascendant de la māya et, comme nous verrons plus tard, le début de l'orgueil et de la conception géocentrique du monde qui, au lieu de considérer l'atmosphère (la place de la tête primordiale) comme le centre de l'univers, déclare (dans sa pensée) la terre comme le milieu du monde.

Dans ce contexte, il faut signaler un fait capital. Alors que le cycle IV est androgyne, le sixième cycle inclut la distinction des deux sexes. Cette évolution s'étend du quatrième au sixième cycle. La vache AUM, placée dans le cycle IV, est une vache-taureau, un être à double sexe. Mais Agni, le dieu du feu, placé dans le monde au milieu des eaux, est également un être androgyne, à la fois vache et taureau. Or le Rg-veda dit que Agni, à peine né, demande du lait à la vache et absorbe la nourriture que la vache elle aussi lui demande. Agni et la vache souhaitent donc la même chose. Un autre passage du $R g$-veda nous aide à comprendre cette situation semi-concrète au bord du monde acoustique. A peine né, Agni saisit le pis du père (AUM) et en tire les rayons et la voix. Agni, le chantre du feu, fait donc résonner et rayonner la sainte syllabe. La vache est la caverne de résonance dont l'activité consiste dans sa passivité, c'est-à-dire dans l'attraction du vide. Encore une fois nous nous trouvons en présence de la pensée antithétique. La caverne de résonance est le symbole du désir d'exister. Dans cette caverne résonante pénètre la voix affamée d'Agni pour se fortifier. Et c'est la même chose, c'est-à-dire la voix, que demande la vache - la caverne - afin qu'elle puisse lui répondre (à Agni). La syllabe AUM du cycle IV est la force maternelle dont la résonance régale d'un lait sonore, c'est-à-dire d'un lait pénétré de la force créatrice et nourrissante.

Transposée dans le cycle VI, la syllabe AUM se prononce d'une autre manière, car dans ce cycle le double sexe est dissous et se répartit en homme et femme. Le dualisme est remplacé par la division en deux éléments opposés. Mais la sainte syllabe garde son caractère dualiste et joue un rôle intermédiaire.

La même situation se retrouve avec Soma (voir plus loin). AUM est la force qui réalise le désir d'exister d'abord d'une manière semi-concrète et semi-acoustique. Son vide, c'est-à-dire sa résonance est la forme d'attraction qui attire et renforce la voix du désir. Et quand le cycle VI introduit le mariage entre deux puissances concrètes, AUM reste abstraite, dualiste et sonore comme autrefois; mais sa voix a changé. Elle n'est plus la voix maternelle; elle est le premier mot 
par lequel les amants se révèlent mutuellement leur amour. Ce mot est, comme nous le verrons plus tard, la parole de l'aurore. C'est le brahman caché qui, d'après l'Atharva-veda, a été trait par la vache AUM, pour que le ciel et la terre, la Vérité et la Non-Vérité, la nuit et le jour puissent se marier.

L'expression «traire la vache» signifie toujours évoquer et obtenir quelque chose par le son, par AUM, c'est-à-dire par le sacrifice. L'action de traire se rapporte aussi bien à une seule personne qui se trait elle-même, qu'à une autre personne, une sentence sacrée, une pierre, la nuit, ou n'importe quel autre objet que l'on trait pour obtenir les bienfaits du sacrifice. On trait, dit le $R g$-veda, le bassin du sacrifice aux quatre ouvertures pour le salut et le bonheur de la vache, et ce bonheur de la vache est également le salut de ceux qui vivent du sacrifice de la vache.

Quelles ont pu être les raisons pour lesquelles on a identifié la syllabe AUM avec la vache? Il est évident que la valeur nutritive du lait a pu la rapprocher de la valeur spirituelle, car AUM est aussi un aliment de l'esprit. Mais on a dû encore avoir une autre raison, ou plutôt une raison parallèle. La suite des sons A, $\mathrm{U}, \mathrm{M}$ aboutissant au silence après l'expiration totale, sert au brahman à suivre son chemin qui conduit son âme du monde concret actuel vers les origines sonores et les tréfonds silencieux de l'ākāça (origine). Or cette formule semble être le produit d'une inversion, technique très courante de la mystique des syllabes. Donc, si AUM mène de la périphérie de la vie vers le centre (vers le monde primordial), son inversion doit être le son créateur allant des origines au monde actuel. Ce son est MUAH, le beuglement de la vache. Les Upanishad confirment notre supposition: $M$ est le ciel, c'est-à-dire le monde primordial; $\mathrm{U}$, le monde intermédiaire, $\mathrm{A}$, la terre.

\section{Le tissage}

Le temps entre l'ascension du soleil après la minuit (IV) et son apparition visible à l'horizon (VII) est la période dans laquelle la matière liquide issue du son se solidifie progressivement. Les principaux symboles de cette matière sont les plantes aquatiques, les cristaux, les cheveux et le fil.

Nous nous occuperons ici uniquement du fil, sortant de la bouche créatrice durant le cycle V. Dans les coutumes populaires, ce cycle correspond aux chambres des fileuses, dont le labeur s'effectue durant la nuit. Dans la mythologie, ce fil est un fil d'araignée, préparant la toile de la māyā. Aussi le créateur du monde concret est-il souvent symbolisé par une araignée.

Le travail de filage terminé, on passe au tissage à la première lueur du jour au cycle VI, afin de créer le monde VII. Ce travail est réalisé par une araignée à six pattes, ou par un métier à tisser où six fils, plus le fil de la navette glissant entre les dents de la bouche, se concentrent dans le cercle VI $1 / 2$, correspondant au III $1 / 2 \mathrm{du}$ monde primordial. Selon l'Atharva-veda, ce furent les sept rși (les rythmes du 7 intérieur) qui reproduisirent cette forme du sacrifice du monde primordial dans le monde concret, pour y créer le septième cycle (VII extérieur). A cette fin, ils 
fixaient les fils au moyen de six piquets enfoncés dans les deux mâchoires, qui sont le ciel et la terre. En faisant basculer ces mâchoires par leur chant, ils tissaient l'aurore à l'aide de sept fils, car la mélodie leur servait de navette. Et ils chantaient des rythmes différents suivant la force ou les dieux qu'ils désiraient créer.

Quand les rṣi tissent et chantent l'aurore au VI⿳1⿴囗十), ils trouvent l'aide de Brihaspati, le «seigneur des chants», qui repousse les ténèbres avec son souffle émis par sa bouche septiforme, et Rohita - soleil matinal appelé «taureau des chants» - étend le tissu de lumière. La aussi, la force provient de la syllabe AUM, tissée dans le panneau par la voix de la vache, dite «vache rouge». C'est elle qui crée le monde visible et tangible. Bien des mythes de création, faisant abstraction des cycles I à VI, ne commencent qu'avec le cycle VII et, conformes au nombre de ce cycle, divisent le temps de la création en sept jours. Parfois on commence aussi avec les ténèbres; dans ce cas, on rattache immédiatement le 7 intérieur (le III $1 \frac{1}{2}$ ) au VII extérieur.

Depuis le début de la création, la syllabe AUM, née du soleil-chantre, a passé trois étapes. Sonore et humide par nature, elle a pénétré les eaux, ensuite le fil, puis le tissu. Et voici qu'elle produit le son-lumière, car c'est elle qui fait monter le soleil. Il y a cependant une curiosité fort intéressante à remarquer. Les mêmes rși, les 7 rythmes primordiaux, qui avec la syllabe AUM ont fait partir le soleil depuis sa base nocturne (III $1 / 2$ ) vers l'horizon, y célèbrent aussi son arrivée. Comment expliquer cette situation? Par la bienveillance des dieux vis-à-vis du monde de la $m \bar{a} y \bar{a}$. Ils prononcèrent les III $1 \frac{1}{2}$ de AUM en 7 temps, créant ainsi le VII intérieur, précurseur du cycle VII extérieur.

Comme le monde, en s'agrandissant de plus en plus, s'était éloigné à tel point de ses origines, le monde primordial est devenu presque imperceptible. On n'entendait plus le son du rta constant ou éternel enfermé dans les cycles III et III $1 / 2$. La lumière du cycle VII «cachait» (pour ainsi dire) le son de la nuit créatrice. On ne voyait plus que le tronc et les branches de l'arbre du monde, et on oubliait ses racines sous terre.

C'est pourquoi les dieux créèrent un nouveau rta VI, VI 1/2, VII, c'est-à-dire une place pour le culte, pour permettre aux hommes un contact avec le monde primordial au moyen d'un plan d'analogie. Ce nouveau rta comprend VI: la libération de la pétrification par le sacrifice, VI $\frac{1}{2} 2$, le mariage de la Vérité et de la Non-Vérité ou du ciel et de la terre pendant l'aurore et, en plus, le rite qui protège les hommes contre le voile de la $m \bar{a} y \bar{a}$ durant la lumière du jour.

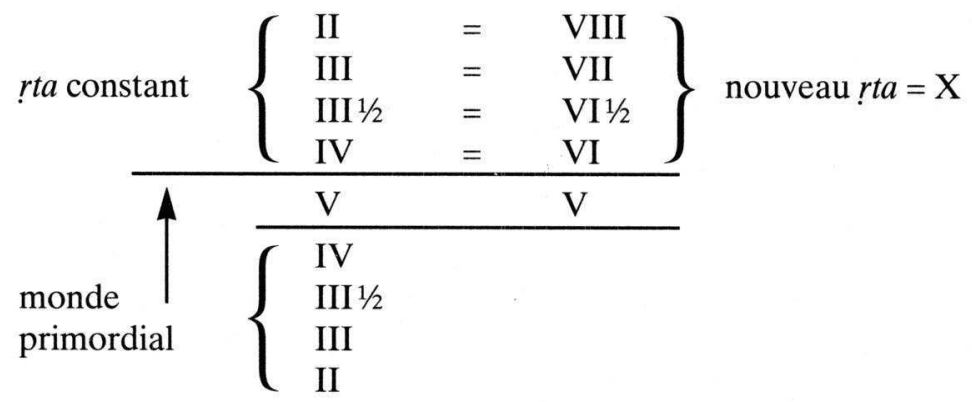


L'aurore permet une vision, bien que voilée et analogue, sur le rta éternel. C'est pourquoi on la considère comme un son-lumière.

Ce rapport entre les deux rta crée une situation qui serait parfaite si les deux rta se superposaient réellement. mais rien que par le rapport d'analogie, ils font entrevoir la perfection, c'est-à-dire le nombre $\mathrm{X}$, car III + VII ou III $1 / 2+$ VI $1 \frac{1}{2}$ font $\mathrm{X}$. Dans le monde primordial, le $\mathrm{X}$ est le nombre égal à 0 , où se trouvent les dix centaines de chevaux dételés du chat solaire, c'est-à-dire la place du soleil immobile, le centre de la Vérité; d'autres versions désignent cette place comme le lieu des dix mille soleils de la région cardiaque du monde. Le $R g$-veda dit que le dieu Indra et les dix mille navaga trouvèrent ce soleil après avoir chanté pendant dix mois dans les eaux. Dix mille chevaux sont dételés dans le rta constant. Et dans le nouveau rta un cheval à sept noms tire la roue solaire munie de trois moyeux, par lesquels passe l'axe du monde.

Musicalement, c'est-à-dire du point de vue de la série harmonique, le rapport III:VII donne une tierce neutre. L'aurore n'est donc pas encore (comme nous l'avons déjà vu) le rapport parfait. Celui-ci ne s'établit que dans le cycle VIII, où le rapport II:VIII donne une octave, c'est-à-dire la consonance la plus parfaite possible dans le monde concret (la dernière perfection serait l'unisson).

Cette extension de la syllabe AUM, siège de toutes les forces, est exprimée par la formule suivante: AUM a 2 têtes, 3 pieds, 4 cornes et 7 bras. Cela veut dire qu'elle a son origine dans le centre dualiste du monde primordial et qu'elle a 3 sons et demi. Ce sont les 3 morae, mouvements de la pensée sonore, plus le son expiratoire. Mais la durée totale, c'est-à-dire son extension dans le monde spatial et temporaire, est de 7 temps. III $1 \frac{1}{2}$ est le solstice d'hiver, VII l'aurore printanière.

Arrivée à ce point, la syllabe AUM touche la fin de son champ d'action nettement perceptible, car avec l'éclosion de la lumière, la connaissance réalisée par la perception du son cède à la pensée abstraite basée sur les impressions optiques. Au lieu de s'approprier les sous-substances de la nature, l'homme du cycle VIII crée des notions et des idées générales qui introduisent la pensée formaliste. Les noms des objets ne sont plus leurs rythmes originels, mais des mots qui désignent ces objets. Les noms ne sont plus des voix créatrices, mais l'expression d'une sorte d'inventaire des objets existants.

Or, pour permettre à l'homme de rester même dans ce monde trompeur proche de la Vérité, le nouveau rta s'étend jusqu'au cycle VIII, c'est-à-dire jusqu'au point où les deux serpents se réunissent de nouveau avec la Vérité centrale (voir fig. 3). Mais cette confluence ne se réalise pas facilement. Elle n'est plus sonore comme l'aurore, elle est purement spirituelle.

Le plus grand danger pour l'homme vivant à la lumière du jour est son égocentricité. Elle est si grande que l'homme déclare même sa terre comme le centre du monde et considère le soleil comme un satellite de la terre. En plus, la tête primordiale étant oubliée, il considère la tête de son Anthropocosme comme le ciel et comme le siège du créateur.

Le soleil n'est plus Ātman, le créateur et l'embryon du centre de l'univers, mais un astre qui se lève et se couche. Il change de nom suivant sa position. Il est successivement Mitra-Varuṇa, Agni, Soma, Savitar, Rohita. Il est Indra quand-il 
atteint son maximum de clarté. Par la $m \bar{a} y \bar{a}$, la vue héliocentrique est devenue géocentrique. Mais c'est la vue conforme à l'homme, et même un cadeau précieux que les dieux ont fait aux hommes. C'est pourquoi le $\mathrm{Rg}$-veda dit: «de voir le soleil et la lune marcher au long du ciel, nous sommes heureux de pouvoir croire en Toi, Indra» (qui conduis le char du soleil).

Le passage entre l'aurore et l'éclosion de la première lumière du jour est le lieu où se produit la «seconde naissance» du soleil et la renaissance rituelle de l'homme, délivré de la pétrification menaçante du cycle VI grâce au nouveau rta.

Le nouveau rta est le centre du chant rituel, ce qui veut dire que le chant rituel relie les cycles III et VII. Il a été démontré que le mot rta, traduit généralement par «ordre» ou «loi», signifie plutôt Vérité, et en particulier la Vérité exprimée par le chant. Mais au fond, il n'y a pas de contradiction entre ces deux traductions parce que le chant rythmé est la force acoustique, fondamentale de l'ordre ou de la loi cosmique.

Le $R g$-veda dit: «Le soleil brille par le chant, né de la Vérité»- «C'est par le chant que nos ancêtres gagnaient leur profonde reconnaissance». La Vérité éternelle est le silence, ou un son à peine perceptible. La Vérité accessible est un son en mouvement. C'est pourquoi les chevaux, traînant le char solaire, sont souvent désignés comme des chants de louange. De même, le timon du char de Sūrya est composé des hymnes.

Dans les rites de sacrifices qui se déroulent pendant l'aurore, le principal propagateur est le dieu Soma. Ici nous trouvons une autre image pour l'activité de la syllabe AUM dans le liquide, à savoir le breuvage sacré offert aux dieux. Soma est une plante qui, écrasée dans un pressoir rituel (VI), coule comme un jus murmurant dans le vase du sacrifice. Pour ce murmure produit par le jus, Soma passe pour être le chantre du sacrifice, ou le sacrifice sonore proprement dit.

\section{Le sacrifice de Soma}

La partie la plus importante du nouveau rta est le sacrifice de Soma, «accompagné», ou plutôt rendu effectif par le chant du Sāma-veda. La plante et dieu Soma, écrasée par deux pierres qui «parlent un rythme sonore», fournit le breuvage qui enchantera le dieu Indra. Le pressoir réalisant le sacrifice est souvent nommé «les dix doigts» ou «les dix rènes qui guident les chevaux» (c'est-à-dire le chant du liquide) vers le récipient du breuvage. C'est au sixième cycle qu'appartiennent les deux pierres du pressoir dans lequel Soma est trituré pour aboutir au VI $1 \frac{1}{2}$ de l'aurore. Pendant cinq jours, les dieux sacrifiaient le Soma, et c'est au sixième jour qu'ils réussirent à le mener à bonne fin.

Le sixième cycle renferme un grand nombre de traits caractéristiques. Je n'en cite que quelques-uns: la création de l'humanité actuelle, l'abolition de la gemellité dans le monde profane, le mariage, la distinction entre les animaux, et le nouvel homme, qui dorénavant sera leur maître, la présence de l'homme au Paradis et sa première chute. Cette nouvelle humanité, qui dès sa naissance se différencie des 
animaux, possède une charpente osseuse considérée comme une pierre. Cette ossification résulte de la première chute provoquée par le serpent de la māa $\bar{a}$, qui introduisit la pesanteur du squelette, l'orgueil de la géocentricité et de l'égocentricité. Mais elle provoque également la pétrification du cœur, de la vie rituelle et de la formalisation ou extériorisation du sacrifice. C'est pourquoi le cycle VI implique la décision de l'homme devant l'alternative ou de rester sensible à son créateur au moyen du sacrifice, ou d'ignorer ces rythmes sonores pour s'adonner totalement à un monde visuel et égocentrique. C'est la décision qui tombe déjà au Paradis : garder un cœur et une oreille ouverts au chant de la grâce ou se fermer en se séparant des sources de vie... et de la joie créatrice (voir le mort qui chante).

Le symbole de cette situation critique est la pierre ou le rocher, qui offre une résistance; le roc est un obstacle. La pétrification signifie: endurcissement, fécondité et concentration, quand elle est pleinement vécue et, grâce au sacrifice courant, surmontée par la libération dans le rite, par l'ouverture de la bouche qui chante. D'autre part, la pétrification définitive symbolise l'orgueil, la vanité et la stérilité, quand elle n'est plus sentie comme un obstacle, mais comme un domicile agréable. Dans ce cas, la gorge reste serrée. Souvent, cette double situation est représentée par une caverne, dont la porte est ouverte ou fermée, résonante ou non-résonante.

Il y a donc dans ce cycle VI une part de culpabilité, qui dans la tradition védique apparaît (entre autres) dans le rapt du breuvage sonore de Soma, dans des calomnies infligées à des personnes innocentes, ou des actes de jalousie, voire des meurtres (asūra, dieux déchus). Tous ces péchés peuvent être lavés par le chant "alala" des fleuves ou par le son-lumière de l'aurore. Le chant est un purificateur de l'âme, les eaux nettoient le corps. C'est pourquoi la purification est un acte caractéristique du sacrifice de Soma. Soma, trituré au son rythmique du pressoir, produit un sifflement et coule ensuite comme un jus sur le filtre placé sur le vase du sacrifice. Ce filtre, qu'il passe en murmurant pour se purifier, est l'aurore, car il existe une étroite liaison entre les mâchoires du pressoir et le métier à tisser. Le tissu est le filtre qui purifie Soma. C'est dans l'aurore que Soma jette les bases pour la nouvelle humanité en ouvrant l'accès aux trois plans de la vie, de la vie sexuelle, végétative et spirituelle.

Dans le neuvième livre du $R g$-veda, il est souvent question de Soma et d'Agni fécondant les vignes, les vaches, les nuages ou les sœurs d'Indra. La vie végétative est assurée par le jus et l'offrande du beurre ou du lait. La vie spirituelle prend son essor dans le breuvage sonore passant le filtre fabriqué dans un tissu ou dans la peau d'un animal de sacrifice. C'est, selon le $R g$-veda, en chantant au rythme du tambour que le prêtre monte sur le «bateau du rta». On dit que ce sont les rși, les mètres poétiques, qui l'expédient vers le vase du sacrifice. Sa voix met tout en marche, comme le rameur fait avancer le bateau.

«Soma, en se sacrifiant (c'est-à-dire en se purifiant et se transformant en breuvage sonore), emporte toutes les fautes». sa force est celle du moment précis auquel il agit. C'est le kaida, le moment juste, ou inhérent à la situation : c'est le moment de l'aurore printanière. Pendant cette heure, Agni et Soma, le feu et l'eau, se croisent et produisent le dialisme fécond: la chaleur humide. C'est le 
dernier moment du dualisme fondamental, car peu de temps après, ce dualisme se dissout en éléments contraires. Agni, la chaleur solaire, descend définitivement sur terre, et Soma, l'humidité, monte comme le brouillard vers le ciel.

Le sacrifice du Soma s'achève quand il se met au service du soleil, c'est-à-dire quand il offre son breuvage sacré au dieu Indra, qui à partir du cycle VII (qui inclut la vision géocentrique) conduit le char du soleil. En se mettant ainsi à la disposition de la lumière, Soma participe à la création du langage articulé et imagé, qui est l'œuvre d'Indra. Nous reviendrons sur cette question du langage, qui en ce moment se distancie de la musique (langage normal). Pour l'instant, achevons le portrait de Soma, dont on dit qu'il est expert en paroles sonores, et même capable de prendre la voix du tonnerre.

Quand on sollicite Soma d'élever sa voix, on lui dit: «Aiguisez la pointe de votre langue», "Mettez votre chant comme une flèche sifflante sur l'arc». A la pointe, c'est-à-dire à la tête du troupeau, allez voir la vache AUM. C'est grâce à la force de la langue triforme de Varuna du monde primordial que Soma transporte ce son sifflant du cycle III au cycle VII.

Ceci faisant, il joue le rôle de l'arbre du monde qui relie tous les cycles par le centre commun de ces cercles concentriques. C'est pourquoi on identifie Soma avec cet arbre, ou on dit que cet arbre - l'axe du monde - sort de son nombril. Que cet arbre (Soma) chante, ressort clairement d'un passage du Çatapata Brahmana disant que l'arbre issu du nombril de Soma sort de la bouche du chanteur. D'autre part, on désigne Soma aussi comme un oiseau qui se pose sur les branches de cet arbre. En fait, Soma est le jus, ou le «lait sonore», dont les gouttes tombent de l'arbre de la vie. Et au pied de cet arbre se trouve la fontaine où jaillit la source qui régale des eaux de la vie. Un jet d'eau monte en résonant à l'intérieur de cet arbre et redescend sur la terre. Soma est le symbole de la circulation des eaux fécondes, bref du cyclose. Aussi est-il appelé le barde, ou le chanteur divin des eaux. En fait, chanter et sacrifier signifient le même phénomène. Et il en est de même pour écouter et boire ou manger. La musique rituelle est un sacrifice, et le fait de l'entendre équivaut à un repas. La musique est le repas des dieux et un aliment pour ceux qui cherchent le contact avec ces dieux. Entendre le chant sacré, c'est boire ou manger la substance acoustique du monde; boire le breuvage sacré, c'est entendre la parole sainte. Le $R g$-veda dit que «les chanteurs touchent le roi Soma avec la bouche. Pour leur salut, les breuvages se réunissent dans un chant de louange». Soma est le patron du chant rituel, appelé Sāma-veda.

\section{Le sāman}

Le sāman est le chant qui donne sa vigueur au sacrifice du Soma. Les assistants au culte y participent par le fait qu'ils unissent leurs voix à celle du Soma et qu'ils boivent le breuvage.

Alors que les textes du $R g$-veda sont récités ou psalmodiés à l'intérieur d'un intervalle de tierce, le sāman est nettement mélodique et se déroule dans un 
espace tonal bien plus ample, organisé par une gamme à sept degrés. Ces deux formes fondamentales, celle du $R g$ - et celle du sāman, accusent toujours un demi-ton, qui pourrait correspondre au passage du cycle VI $1 / 2$ au VII. (Mais c'est une supposition, pour laquelle je n'ai pas de document.) Par opposition au $R g$ veda, dont la récitation suit le mètre poétique, le sāman accuse un rythme bien plus complexe. A côté des textes originaux, souvent presque ou totalement incompréhensibles, le sāman se sert aussi des textes du $R g$-veda, bien que parfois extrêmement déformés. En plus, il intercale - sans égard pour les données sémantiques ou grammaticales - des syllabes sonores nouvelles, ou des voyelles tirées du texte, mais longuement prolongées. Par ce procédé, la force sonore enfermée dans les mots du langage normal est libérée et répartie sur une longue ligne mélodique. La syllabe AUM se place généralement au début et à la fin du chant. Parfois intercalée, ou plutôt suggérée au milieu du chant par un léger murmure, elle doit causer l'identification du chanteur avec le plus haut brahman. Le sāman est un bateau qui conduit vers les dieux, toujours sonores.

Sa musique constitue un répertoire de formules, toujours répétées d'une façon plus ou moins analogue au cours d'un chant. On adapte le texte à ces formules préexistantes. La musique est donc prédominante. Pourtant ce flux mélodique est parfois interrompu par des interjections, plus ou moins semblables aux éléments du langage syllabique. Mais dans son ensemble, le sāman est une sorte d'amplification des sons mystiques, liés entre eux pour former un ensemble mélodique, en s'appuyant sur la suite des voyelles du texte, pour mettre leur sonorité en valeur.

Par opposition au langage des syllabes mystiques, le sāman n'imite pas les bruits de la nature. Sa structure fondamentale se déroule dans un réseau tonal divisant l'octave en 22 parties, appelées çrūti. Et à l'intérieur de ces 22 çrūti, on choisit une gamme de 5,6 ou 7 degrés bien distincts. Or çrūti veut dire « révélation divine». Le nombre 22 se retrouve aussi dans d'autres cosmogonies. Il est probable que - de même qu'ailleurs - ce nombre se compose de $3 \times 7+1$ (encore 3 et 7 , mais multipliés. 21, nombre total des 10 cercles doubles).

Le Çatapata Brahmana dit que le langage de l'univers se compose de quatre parties, et que ce n'est qu'un quart de ce langage qui est propre aux hommes. Selon d'autres traditions, un quart appartient aux dieux, un autre aux morts, un troisième aux hommes et le dernier quart aux animaux. La Chāndogya Upanishad souligne que les voyelles viennent d'Indra, les sifflantes (Ç, S et S) et aspirées $(\mathrm{H})$ de Prajāpati, les mutuae, c'est-à-dire toutes les consonnes, appartiennent aux morts. Parfois on attribue aussi à chacun des sept sons des dieux, les hommes, le bétail, les herbes; mais ces traditions sont très contradictoires. Ce qui est sûr, c'est qu'on cherchait à concentrer dans ces sept sons l'ensemble de l'univers, en soulignant la grande participation des dieux et des morts, et son aboutissement au dieu de la lumière, Indra.

Bien que le chant du sāman ne se plie pas au mètre poétique des textes, il adapte quand même son rythme. D'après le $R g$-veda (I, 144), ce rythme constitue la base dont le mètre a pu être déduit. Le rythme est primaire, le mètre est une schématisation secondaire, tirée du chant. La base rythmique est fondée par 
certains types de mouvements qui se reflètent probablement dans les $v r t t i$, que la littérature moderne appelle «rythmes oratoires». Le sāman est une combinaison entre les rythmes naturels et les mètres réglementés de la poésie (positin intermédiaire aurore-lumière, chant-langage).

Les différentes formules constituant les élans rythmiques du säman ont une importance capitale, parce qu'ils incarnent les membres, ou même la colonne vertébrale du créateur Prajāpati, qui avec son fils Soma forme l'arbre du monde, ou l'axe de l'univers. Leur essence est toujours la même. Les premiers dieux et les premières offrandes de sacrifice étaient des rythmes.

Le Çatapata Brahmana nous a conservé un grand nombre de ces rythmes fondamentaux. A l'énumération de leurs noms elle ajoute pour chaque mètre l'objet visé et la façon d'agit sur cet objet. Par exemple: l'Infini est touché par le rythme appelé «océan» ou «chant de louange»; les aliments sont atteints par le rythme «avaler» ou «investir»; le souffle est touché par un rythme appelé «fleuve», ou "la terre", qui correspond à la Gāyatrī composée de $3 \times 8$ syllabes. Le ciel est contacté par le mètre «Bienheureux» de 23 syllabes; Indra par triștubh de 44 syllabes, etc. (nature du rythme = sa fonction!).

Malheureusement, les rythmes avec lesquels ces mètres furent lancés n'ont pas pu être communiqués, car leur réalisation ne peut se transmettre qu'oralement, c'est-à-dire directement par le gourou à l'élève.

Il nous reste cependant quelques indications sur certaines pratiques. D'abord, il faut que le chanteur commence à organiser les mélodies et les textes selon leur rythme, c'est-à-dire d'après leurs fonctions. Ensuite, on recommande de changer souvent de mètre, parce que les mètres - de même que les animaux traînant un char - se fatiguent. Pour atteindre un dieu (grâce au rythme qui lui convient), on commence par chanter seulement le premier quart du vers. Puis on recommence en poussant jusqu'à la moitié du même vers. Après avoir repris le vers pour la troisième fois, on va avec plus d'élan jusqu'aux trois quarts, dont la fin touche la frontière du règne des dieux. Une autre règle dit: on avance considérablement avec sept vers, mais avec le nombre 7 on n'arrive pas jusqu'au bout. Avec 9 vers, on pénètre dans le monde intermédiaire; avec 10 vers, on surmonte le dernier obstacle.

Ce dixième vers doit s'évanouir dans le silence, parce que 10 est égal à 0 . Par ce dixième son, on passe au son inaudible et immortel appelé năda. Comme chant audible, le sāman ne s'étend donc que du cycle III au cycle IX. Au cycle VII (aurore), il est nommé l'utérus maternel des dieux. On rapporte qu'à cet endroit, c'est-à-dire pendant l'aurore, ces dieux, nés comme des mortels, ont vu le săman de leurs propres yeux. Alors ils réfléchirent de quelle manière ils pourraient devenir immortels. Et, après avoir fait des austérités, ils virent les chants et ils se mirent à les chanter; et voici que leurs os disparurent et qu'ils prirent le chemin de l'immortalité. Cela veut dire qu'en passant du cycle VII au VI qui renferme la pétrification ou l'ossification, ils s'engagèrent par $\mathrm{V}$ et IV sur le chemin menant au monde acoustique primordial, qui est en dehors de l'espace et du temps, donc immortel.

Selon une autre version, ces dieux entendaient le lait, c'est-à-dire le souffle sonore, de la vache AUM. Faisant des sacrifices, ils se rapprochaient du pis de la 
vache. Après un certain temps, ils voyaient seulement 3 tettes, ensuite plus que deux, et finalement une seule. Cela veut dire qu'au début, ils voyaient et entendaient les 3 sons A-U-M, ensuite seulement U-M, et finalement il ne restait que le $\mathrm{M}$. A ce moment, ils se sentaient si proches de la réalisation de leurs vœux qu'ils renonçaient même au lait, c'est-à-dire qu'ils expiraient par le son S. Et quand ils cessèrent de respirer, ils devinrent immortels.

Cesser de respirer veut dire: voir l'Éternité après la mort; et si le yogi prononçant AUM arrête sa respiration durant ses méditations, il accomplit un acte d'analogie.

Le sāman, pénétré du souffle de la vache AUM, est le chemin vers la mort, suivie de l'immortalité si le mourant expire avec la sainte syllabe sur les lèvres. Par elle, il entre dans la substance sonore de l'univers qui, en devenant inaudible, devient immortelle. Ce sāman, dit le Çatapata Brahmana, est la Vérité.

\section{A UM-A MEN}

Pour devenir immortels, les hommes prennent donc le même chemin que les dieux nés pendant l'aurore (qui ne sont pas l'éternel àtman, éternel et inaudible). Sous ce rapport, ces dieux nés pendant l'aurore sont liés aux hommes par la base commune constituée par le son. Le moment de leur naissance coïncide même avec la position cosmogonique de la naissance des hommes, ce qui établit même une sorte de parité entre les dieux nés mortels et les hommes. Mais comme ces dieux ont gagné l'immortalité avant les hommes, ils deviennent une sorte de saints, d'intercesseurs, dont les rythmes sont accessibles aux mortels.

D'autre part, ces dieux, étant des rythmes sonores, ont aussi besoin des hommes. Bref, il y a une interdépendance acoustique.

On dit que quand les dieux ont épuisé la force de leurs rythmes, les chanteurs terrestres doivent venir à leur aide. Alors les mètres terrestres volent comme les oiseaux vers les dieux qui ont soif. Au lieu des oiseaux, on cite aussi des vaches ou des bêtes de somme qui, traînant le char du sacrifice vocal, secourent les dieux. Le chant des mortels nourrit les dieux. Voici quelques passages du $R g$ veda: «- Avec l'offrande à la main et le chant dans la bouche, nous secourons les dieux. - Que mon chant soit un aliment nourrissant pour Indra. - Mon chant est plein de beurre et de miel. Il est une viande exquise pour le dieu affamé».

Un chant de louange fait littéralement grandir le dieu auquel il est adressé. Mais tous ces dieux sont les dieux de la $m \bar{a} y \bar{a}$, nés pendant l'aurore, au point de rencontre de la Vérité et de la Non-Vérité, ils sont les saints du monde concret. Le dieu du monde acoustique est dans le monde primordial. Néanmoins, la relation entre le monde primordial, les dieux de l'aurore et les hommes repose essentiellement sur l'audition. Le siège de Brahmā est dans l'oreille.

Une condition sine qua non est la continuité du sacrifice sonore. Il faut qu'il soit ininterrompu comme la colonne vertébrale, car la moindre fissure ou la moindre interruption de la respiration peut permettre aux démons de se glisser dans le flux du rythme. 
La position cosmogonique du sāman s'étend du cycle V au cycle VII, tout en étant pour ainsi dire suspendu au III et au IX. De cette position est dérivée l'organisation de ses formes: la répétition ternaire des formules fondamentales et la division d'un chant en 5 ou 7 parties. Ces différentes phases sont associées principalement aux 5 ou 7 étapes de la marche du soleil et aux plans de la vie attribués à ces différents stades (dieux, saisons, âges, etc.).

Le sāman est la formule sacrée élevée à son plus haut niveau. Il est l'essence du sacrifice, parce qu'il est le plein consentement à l'offrande du souffle vital par le son. Les deux formes les plus vénérées, le rathāntara et le brhat, sont les formules secrètes qui - telles un bateau à voiles - se dirigent vers les dieux. Mais là encore, le rôle de l'homme est de la plus grande importance, car quand le prêtre prend le vase rempli du breuvage sacré entre ses mains, c'est le chanteur qui y met la force. L'enthousiasme de Soma, la sainte volonté des sacrifices, provient de l'enchantement que la mélodie humaine lui confère (Rg-veda IX, 71, 3).

\section{Les cakra}

Notre hypothèse sur le son Ș, partant de la pointe de la langue de Soma, est confirmée par la doctrine des cakra. Cette doctrine, pratiquée par les yogis, divise le corps humain (considéré comme microcosme) en 7 centres, lotus ou cakra, qui correspondent aux cycles III à IX. Chaque centre (cakra) est le représentant d'un ou de plusieurs organes du corps et d'une série de sons, exprimés par les lettres de l'alphabet. Le point de départ est le parabindu, un point géométrique inaudible de triple nature formant une unité. Ce parabindu, en se fendant, se manifeste: 1 . comme năda, qui est un son indifférencié ; 2 . comme bindu (expiration nasale); et 3 . comme bija (racine des consonnes). Ces trois données élémentaires se situent au cycle III, constituant le triangle équilatéral nommé le triangle du vœu divin de créer le monde.

Les 3 sons correspondants sont A-KA-THA du monde primordial. Chacun des 3 côtés du triangle renferme 16 sons, et dans chaque angle se trouve encore un son. Cela fait 51 sons. La série des 16 sons du premier côté du triangle, portant 16 voyelles, commence avec la lettre $A$, le deuxième côté commence avec $K$, le troisième avec TH, = AKATHA. A est la forme acoustique du larynx, KA du cour et THA du nombril. Voici le monde souhaité par le créateur: le triangle AKATHA formant un nombril, un cœur et un larynx, et dont le créateur luimême est la tête contenant cette idée sonore.

Or, dès que la volonté d'exister (mānas) se manifeste, nāda (le son indifférencié et originel) se manifeste en forme de spirale sonore, symbolisée par un double serpent nommé kuṇdalini. Enroulé dans le sommeil primordial, il se lève au cycle III $1 / 2$ avec un sifflement $S$, correspondant au cakra du coccyx (de l'Anthropocosme), et atteint, après avoir traversé en ligne hélicoïdale le sexe, le cœur et le larynx, la pointe de la langue. Arrivé à ce point, le front et le nez lui opposent le son spirituel $\mathrm{H}$, sur quoi le serpent se retire et retombe dans son sommeil. 
Ce serpent est un être double en tant qu'il monte et descend autour de la suṣumṇa, sorte de colonne vertébrale idéale ou arbre du monde. C'est le bâton de Mercure. Ce bâton, souvent invisible, est la Vérité absolue qui ne connaît pas de forme. Le serpent est la vérité relative et dualiste de la māy $\bar{a}$, qui tourne autour de la Vérité en créant le monde matériel des formes. Issu de la même racine que la Vérité, il se sépare d'elle au coccyx et y revient dans la région du nez, si elle abandonne le monde matériel. Sinon, elle redescend au point de départ.

Cette image nous montre le double aspect de Brahman. La Vérité est le brahman comme formulation sonore; la Non-Vérité est Brahmā conçu comme un dieu corporel, c'est-à-dire comme une représentation visuelle d'un phénomène qui dans sa véritable nature est purement acoustique.

Ces deux aspects de brahman sont deux états de conscience. Dans le premier, l'homme dormant d'un profond sommeil est englobé dans la vie cosmique et, par conséquent, sa conscience reste limitée à la perception des formes. Il se constitue en individu indépendant et s'établit en observateur de la nature. Ceci se produit quand kuṇ̂lalinī le serpent «ferme la porte» de la Vérité absolue. Cette porte est située au cycle $\mathrm{III} \frac{1}{2} 2$, au passage du monde acoustique à la création concrète. C'est là où Varuna, le dieu de l'océan primordial, place sa langue triforme, dont on dit que celle du milieu est presque insaisissable.

Voyons maintenant la disposition des lettres de l'alphabet. D'abord quelques remarques préliminaires: la consonne $\mathrm{M}$ passe pour être androgyne. Elle s'applique et aux yeux et au sexe. Le coccyx comprend aussi les parties génitales extérieures.

\section{Les cakra ou centres}

3. Cycles I-III: la tête primordiale qui renferme le triangle du plan divin A-KA-THA - larynx, cœur, nombril.

Par la suite se produit le sifflement du serpent créant le monde concret 6.7. à gauche. III $1 \frac{1}{2}$, coccyx.

5. Situation au moment de l'aurore (représentation classique des cakra du yogi en méditation). A partir du cycle III 1/2. Le centre IV, V, VI, 10.

6.7. Les voyelles et les consonnes correspondantes.

8. Solstice, équinoxe.

6. La répartition des lettres de la syllabe AUM.

La voyelle originelle est le $\mathrm{O}$ qui occupe le centre, c'est-à-dire le cœur. Cette voyelle $\mathrm{O}$ est la voyelle génératrice, qui en se fendant (tel le parabindu) produit le A et le U. Quand kuṇdalinī se réveille avec le son Ș (7. à gauche), elle passe toutes les consonnes, c'est-à-dire les cakra depuis le coccyx jusqu'au cœur, donc en partant des labiales pour aboutir aux gutturales. Au cœur, les sons intérieurs commencent à devenir perceptibles. Puis, en passant par la semi-voyelle Y, kuṇ̂lalinī arrive à la porte de la libération, c'est-à-dire à la bouche où se produịsent les voyelles. Ici se réalise le tissu de l'aurore. Et le S sur la pointe de la langue rencontre le $\mathrm{H}$ issu du nez.

Dans le chant rituel, les consonnes ou les voyelles mises en relief sont toujours essentiellement celles du cycle sur lequel on désire agir. 
Quand les voyelles sont acquises, tout le corps devient plus sonore. Les consonnes obtiennent un corps. K devient KA, TH devient THA, etc. La formule AUM se répartit de la façon suivante (6.). Elle est AUM (Ș) lorsqu'on se dirige de la $m \bar{a} y \bar{a}$ vers la Vérité; elle est Ș-MUOA quand elle crée le monde.

La bouche, le centre de libération au moment de l'aurore, est le centre du nouveau rta. Sa position VI_correspond au III $1 \frac{1}{2}$. Cette relation a donné lieu aux deux types de mythes de création. L'un commence avec III $1 \frac{1}{2}$, c'est-à-dire avec le solstice d'hiver (8.), l'autre avec le printemps, où dans le cycle VII se réalise la création en 7 jours.

Une position de ce genre occupe le livre de Moïse, et également le dieu égyptien Thot qui, après avoir ri sept fois sur le son guttural KHA (7.), le son du cœur, passe à la semi-voyelle Y pour énoncer les voyelles de l'aurore: YAO!

Mais ce cycle VII créateur implique le 7 intérieur. Le KHA est aussi le son du cœur du monde primordial, cet espace entre III $1 / 2$ et VII apparaît aussi dans le livre de Moïse. Dieu prononce d'abord la lumière. Cette lumière sonore ne peut pas être la lumière solaire, puisque le soleil est créé seulement au troisième jour. La lumière sonore initiale est celle du rta divin: III $1 \frac{1}{2}$ ou 7 intérieur. Les 7 jours de la création sont la matérialisation des sons harmoniques 7 à 14, qui troublent les sons 4 à 7 par l'octave, et divisent les cycles en deux.

\begin{tabular}{|c|c|c|c|c|c|c|c|c|c|}
\hline Cycles et sons harmoniques & & IV & & $\mathbf{V}$ & & VI & & VII & \\
\hline Sons harmoniques & 7 & 8 & 9 & 10 & 11 & 12 & 13 & 14 & 15 \\
\hline Jours de la création & 0 & 1 & 2 & 3 & 4 & 5 & 6 & 7 & \\
\hline \multicolumn{10}{|l|}{ 0. Lumière sonore du rta } \\
\hline \multicolumn{10}{|l|}{ 1. Le ciel } \\
\hline \multicolumn{10}{|l|}{ 2. La terre } \\
\hline \multicolumn{10}{|l|}{ 3. Le soleil } \\
\hline \multicolumn{10}{|l|}{ 4. Poissons et oiseaux } \\
\hline \multicolumn{10}{|c|}{ 5. Animaux terrestres (serpent) } \\
\hline \multicolumn{10}{|l|}{ 6. Homme au Paradis } \\
\hline 7. Soleil à l'horizon & & & & & & & & & \\
\hline
\end{tabular}

Revenons aux cakra. Le cakra du front porte essentiellement le son $\mathrm{H}$, un $\mathrm{S}$ très sonore et KṢ. Les yeux et les oreilles sont - de même que le sexe - par le son androgyne M. Prononcés d'une manière nasale, $\mathrm{H}$ et KȘA donnent Hamsa, ou son renversement So-Ham. Quand on émet cette formule, on arrête la respiration entre les deux syllabes. Ce moment d'arrêt signifie: je suis Lui, je suis Brahman.

Dans le langage courant, le mot Hamsa signifie le cygne ou l'oie, parfois même le canard. Mais l'idée prédominante est le cygne.

Ceci nous amène à jeter un regard sur les étoiles correspondant aux cycles III $1 / 2$ à VII (9.). Les constellations zodiacales sont:

\begin{tabular}{cccc}
\hline IV & $\mathbf{V}$ & VI & VII \\
Aquarius & Piscis & Aries & Taurus \\
\hline
\end{tabular}


Au cercle polaire (III, III $1 \frac{1}{2}$ ), se trouvent le Cygne et la Grande Ourse (le VII intérieur) du printemps. Non loin de lui brille Orion à 7 étoiles, et dans le Taureau même, les Pléiades, que l'on désigne comme une constellation à sept étoiles, bien qu'en vérité elle en possède seulement 6 .

Or, selon la tradition, la septième étoile est celle qui à présent se trouve sur le timon du Grand Chariot (Grande Ourse). Elle a quitté le VI $1 \frac{1}{2}$ pour aller vers le III $1 \frac{1}{2}$. Souvent, les Pléiades sont nommées la maison de chasseurs où habitent des filles poursuivies en vain par le grand chasseur Orion. La mythologie grecque nous raconte que Callisto la Grande Ourse, après avoir perdu sa virginité par l'intervention de Jupiter, a été reléguée par Artemis du grand océan à l'océan primordial, c'est-à-dire à sa place actuelle au III $1 \frac{1}{2}$; et de cette place, elle regarde sans cesse vers Orion. Dans d'autres contes, un chasseur fourvoyé arrive au lac des cygnes du monde primordial (cercle polaire). Il voit une jeune fille au bain, lui vole son vêtement de plumes de cygne et l'oblige à l'accompagner chez lui et à se marier avec lui. Mais, après avoir donné la vie à un enfant (cycle VI 1/2), elle quitte son mari pour rentrer au lac des cygnes. De même, le cygne de Brahmā au cycle III réapparaît comme Indra au septième cycle (encore III-VII). Mais, alors que le cygne Indra continue sa route, le cygne Brahmā se retire pendant l'aurore au cycle III.

Par ces deux cygnes, nous pouvons préciser la signification de l'aurore. L'aurore - la position cosmologique de la musique sacrée donnant une vue voilée sur le rta éternel - est le mariage entre le ciel et la terre, entre la Vérité et la Non-Vérité, symbolisée par les deux cygnes, c'est-à-dire par le Brahmā de la Vérité et le brahman de la $m \bar{a} y \bar{a}$, représenté par Indra et le char solaire. Ce mariage a été préfiguré par le soleil séduisant l'aurore pour favoriser le développement de cette $m \bar{a} y \bar{a}$. Et quand cette fête printanière a pris fin, la future mère (l'aurore) se retire au règne de la Vérité pure, nocturne et sonore, et c'est au solstice d'hiver qu'elle met au monde son enfant, c'est-à-dire le nouveau soleil. Cette mère (au Rg-veda la troisième Ușā) est le cygne noir, le chanteur qui se retire au Septentrion pendant qu'Indra, le père, est le cygne blanc qui continue sa route en traînant le bateau du soleil au long du ciel. Accompagné d'abord des marut, il fait partie d'un groupe de sept cygnes.

Tout cela est l'œuvre d'Indra, un mélange de Vérité et de Non-Vérité, de sons et d'images, de chants et de paroles. Son action est Satyam. Sa et Yam sont la Vérité, alors que $T$ est la Non-Vérité, mais dominée et enfermée par la Vérité.

\section{Le langage rituel}

Étant donné que l'univers s'élève sur une base acoustique, $v \bar{a} c$, la voix, est un organe indispensable à la réalisation du monde. Mais $v \bar{a} c$ est une «personne» absolument neutre, nous dit la mythologie. Elle n'a pas d'opinion, elle ne prend jamais position. Elle n'est qu'un véhicule de la pensée, car la force n'est ni la voix, ni le mot qu'elle prononce. La force est AUM, qui chevauche la voix. Aussi 
une lutte acharnée s'est-elle livrée entre les dieux et les démons pour gagner la faveur de cette personne $v \bar{a} c$, issue des eaux sonores de l'océan primordial (bouche). Sans elle, aucun sacrifice essentiel, c'est-à-dire sonore, n'est possible; et si les dieux ont fini par gagner la bataille, c'est parce que les démons, leurs adversaires, n'utilisaient pas la voix avec le dévouement sacrificiel nécessaire, mais avec orgueil. Le Çatapata Brahmana dit que, orgueilleusement, ils sacrifiaient dans leur propre bouche.

Un jour, $v \bar{a} c$, gagnée par les dieux, épousa le sacrifice, et de cette union naquit Indra, le dieu du jour. D'autres traditions disent que Sāma-Veda mâle épousa $R g$ veda femelle, et que de ce mariage du chant et du langage naquit Indra, qui à son tour créa le langage bien articulé, clair et distinct.

Nous voici devant un autre aspect du langage sacré. Nous avons vu qu'Indra est le dieu du monde géomorphique, qui crée le soleil à l'horizon et élargit l'espace de l'univers grâce à la $m \bar{a} y \bar{a}$. Aussi le vocabulaire de son langage n'exprime plus l'essence rythmique des objets, mais se limite à les désigner. La pensée rythmique et musicale a cédé le pas à la pensée logique et formaliste, créée pour une humanité qui s'oriente essentiellement par la vue.

Mais, conscient de cet obscurcissement de la réalité ou de la Vérité par la Non-Vérité, ce langage (surtout dans les données mythologiques) a souvent un double sens, ou implique une limitation de ce qu'il dit.

Il est vrai qu'Indra est un combattant tout-puissant... mais l'a-t-on jamais vu? A-t-il vraiment des adversaires? On ne répond pas avec non, mais on pose quand même la question. Et après l'avoir mise en doute, on dit : «En comparaison avec la force d'Indra, tous ces adversaires ne comptent pour rien ». La situation reste entre la Vérité et la Non-Vérité, mais cette fois-ci comme un jeu de balance.

Souvent ce langage ne dit ni oui ni non, car la Vérité de notre langage clair et distinct n'est jamais plus que la moitié de la Vérité. C'est parce que la Vérité entière ne peut être exprimée avec des mots de notre langage formaliste.

C'est pourquoi il faut que le langage, pour rester rituel, tienne toujours compte des étapes précédentes, c'est-à-dire du rythme musical et des eaux sonores dans lesquels il est enraciné. Ceci dit, nous pouvons noter neuf traits caractéristiques du langage rituel:

1. Des symboles optiques indiquant des phénomènes sonores (la caverne comme fond sonore, le fils ou la fille du créateur représentant la voix du créateur; le char ou le bateau, un oiseau, une vache ou une flèche pour le chant; boire ou manger pour entendre; sacrifier pour chanter; le verbe traire signifie obtenir quelque chose au moyen de a voix).

2. Les nombres associés à un phénomène caractéristique pour un cycle donné (sept chevaux, sept bouches, sept mains).

3. Les mots homonymes ont la même signification fondamentale ou procèdent l'un de l'autre, bien que leur valeur sémantique soit complètement différente (svara $=$ son, svar $=$ lumière $)$.

4. L'entrelacement logiquement incohérent des plans visuel et intellectuel. Par exemple: «le tissu de la vérité sonore étendue sur le filtre» ou «sur la pointe 
de la langue », ce qui veut dire que la Non-Vérité purifiée par le son est devenue audible grâce au jus du soma durant l'aurore.

5. Quand la fonction d'un objet varie au cours du temps, cet objet reçoit un autre nom exprimant la variation de cette fonction. Avant de paraître à l'horizon, le soleil est un poisson, en se montrant à l'horizon il est un veau, puis un taureau ou un cheval.

6. Le flux rythmique a une telle importance que les chants doivent être classifiés selon les mètres.

7. Dans leur essence, les hymnes sont des chants de louange, dont la musique nourrit les dieux.

8. La substructure du langage sacré est essentiellement musicale, et c'est de là que procèdent ses formules linguistiques parfois tellement énigmatiques. En principe, le langage rituel védique cherche à s'exprimer simultanément sur deux, sinon trois plans différents.

9. De là s'explique le caractère ambivalent de ses expressions.

Ce langage kaléïdoscopique est la conséquence de sa substructure multiforme résultant de la superposition des différents cycles. On aurait tort de vouloir l'expliquer par un plaisir à la cryptomanie. Il est possible que, conformes à la nature des cycles IV à VI, beaucoup de formules soient des reflets d'images perçues dans le rêve. En plus, il est normal que l'idée de «traire la vache» pour «obtenir la véritable nourriture» soit toujours présente chez un peuple d'éleveurs de bétail.

D'autre part, la réunion de plans hétérogènes doit obéir à une intention bien définie. Prenons quelques exemples: «étendre le fil de la Vérité sur le filtre» ou «la vache dont le veau est le soleil, la corde d'attache un mètre poétique, le pis un nuage, les tettes des chants». Une telle combinaison de plans différents sert tout simplement à faire sortir la Non-Vérité ou l'irréalité du monde de la māay, formulé par notre langage formaliste. Ces associations d'images hétéroclites mènent la pensée qui, tout en étant abstraite, se réalise au moyen d'images et de notions générales. Elles cherchent à réduire les deux extrêmes: la pensée abstraite pure d'un côté et la conception optique du monde concret de l'autre, à un plan intermédiaire, c'est-à-dire au monde intermédiaire du culte, qui est le chant.

Il n'est donc pas étonnant que la pratique religieuse aboutisse finalement toujours au chant, même quand elle récite des textes en langage formaliste, car ce texte ne peut devenir vrai que lorsqu'il est chanté. On dit que même les événements du passé récités dans les épopées revivent et redeviennent vrais quand ils sont chantés. La présence de la Vérité ne peut être conçue que par le rythme sonore pur de la musique, car le langage, qui est bien plus forme que substance, est incapable d'exprimer un état de choses qui, par nature, n'a pas de forme.

Aussi le yogi qui, pour obtenir le contact avec la Vérité, élimine toute pensée et toute représentation visuelle poursuit dix degrés de méditation. Les deux premiers degrés sonnent cini et cinini, le troisième est un son de cloche qui fatigue, le quatrième un son de conque marine qui inspire des doutes vis-à-vis de notre monde concret. Le cinquième son provient d'un instrument à cordes qui irrite le 
palais. Au sixième degré on bat dans les mains, ce qui fait boire au yogi le monde suprasensible. Par le son de la flûte, le septième degré lui transmet la connaissance ésotérique. Au huitième degré, un tambour lui inspire le langage sacré. Par un autre tambour, il acquiert l'invisibilité au neuvième degré et, finalement au dixième degré, un coup de tonnerre l'introduit dans la Vérité absolue de Brahmā.

\begin{tabular}{|c|c|c|}
\hline Sommet de la tête: & passage $S$ & $\mathrm{H}$ inaudible \\
\hline Front-œil: & $\begin{array}{l}\mathrm{Ks} \\
\text { bija } \longrightarrow\end{array}$ & $\begin{array}{l}\mathrm{H} \\
\text { AUM }\end{array}$ \\
\hline Larynx: & $a \bar{a} \mathrm{i} \overline{1} \mathrm{u} \overline{\mathrm{u}} \mathrm{r} \overline{\mathrm{r}} \mathrm{lr}$ & $\bar{r}$ e ai o au am ạ̣ \\
\hline Cœur: & $\begin{array}{l}\text { gutturales } \\
\text { palatales } \\
\text { cérébrales } \\
\text { bija } \longrightarrow\end{array}$ & $\begin{array}{l}\text { k kh g gh n } \\
\text { c ch j jh n } \\
t \text { th } \\
y(j)\end{array}$ \\
\hline Nombril: & $\begin{array}{l}\text { cérébrales } \\
\text { dentales } \\
\text { labiales } \\
\text { bija }\end{array}$ & $\begin{array}{l}\text { d dh }{ }^{n} \\
t \text { th d dh n } \\
\text { p ph } \\
R\end{array}$ \\
\hline Sexe: & $\begin{array}{l}\text { labiales } \\
\text { semi-voyelles } \\
\text { büja } \longrightarrow\end{array}$ & $\begin{array}{l}\mathrm{b} \text { bh m } \\
\text { y } \mathrm{r} \mathrm{l} \\
\mathrm{v}\end{array}$ \\
\hline Coccyx: & $\begin{array}{l}\text { semi-voyelle } \\
\text { sifflante palatale } \\
\text { sifflante cérébrale } \\
\text { dentale }\end{array}$ & $\begin{array}{l}\mathrm{v} \\
\mathrm{c} \\
\mathrm{s} \\
\mathrm{s}\end{array}$ \\
\hline Passage & $\mathrm{S} \longrightarrow$ & $\mathrm{H}$ inaudible \\
\hline
\end{tabular}

Répartition des lettres dans les cakra. 


\section{Bibliographie}

Autres publications de Marius Schneider sur la cosmologie et le symbolisme sonore. Bibliographie établie par Robert Günther ${ }^{1}$.

1949 "Consideraciones acerca del canto gregoriano y la voz humana», Arbor 14 (48): 367-84.

1950 «Vom ursprünglichen Sinn der Musik», in: Bericht über den Vierten Kongreß der Internationale Gesellschaft für Musikwissenschaft, Basel 1949. Basel.

1951 «Die historischen Grundlagen der musikalischen Symbolik», Die Musikforschung 4: 113-144.

1952-53 «Die Bedeutung der Stimme in den alten Kulturen», Tribus (Stuttgart) 2/3: 9-29.

1955 Singende Steine. Rythmus-Studien an drei katalanischen Kreuzgängen romanischen Stils. Kassel: Bärenreiter ( $2^{\mathrm{e}}$ éd. augm. München, 1978).

1959 «Origin of the symbol in the spirit of music», Diogenes 27:39-62.

1960 «Le rôle de la musique dans la mythologie et les rites des civilisations non européennes", in: Roland Manuel (éd.) Histoire de la musique I (Encyclopédie de la Pléiade): 131-214. Paris: Gallimard.

1960a «Zur Bedeutung der Flügel», in: Museion. Studien aus Kunst und Geschichte für O.H. Förster: 50-57. Köln.

1960b «Sociologie et mythologie musicales», in: Les Colloques de Wégimont III-1956. Ethnomusicologie II: 13-22. Paris: Les Belles Lettres.

1960c «Die musikalischen Grundlagen der Sphärenharmonie», Acta Musicologica 32:136-151.

1960d «Musik (II); Religionsgeschichtlich", in: Die Religion in Geschichte und Gegenwart Bd. 4 ( $3^{e}$ éd.): 1197-1201. Tübingen.

1961 «Die Harmonie der Sphären», gehört-gelesen 1:28-33.

1962 «Das Morgenrot in der vedischen Kosmogonie», Symbolon 5: 61-75.

1964 «Die Natur des Lobgesangs», Basilienses de Musica orationes H. 2. Basel: Bärenreiter.

1964a «Jucunda laudatio», in: Rassegna gregoriana II: 80ff. Venise.

1967 «Pukku und Mikku. Ein Beitrag zum Aufbau und zum System der Zahlenmystik des Gilgamesh-Epos", Antaios 9: 262-283.

1968 «Le symbole sonore dans la musique religieuse ou magique non européenne», in: Jacques Porte (éd.) Encyclopédie des musiques sacrées vol. 1: 53-79. Paris: Labergerie.

1970 Il significato della musica. 2e éd. Milano: Rusconi.

1970a «La notion du temps dans la philosophie et la mythologie védique», in: I valori permanenti nel divenire storico. Instituto accademico di Roma. Firenze: Vallechi.

1971 «Natur und Ursprung des Symbols», Zeitschrift für Ganzheitsforschung (Wien).

1973 «La coppia simbolica 'musica e pietra'», in: Conoscenza religiosa.

1973a «Die Grundlagen der Kultsprache in der vedischen Kosmologie», in: Sprache und Sprachverständnis (hrsg. v. Th. Michels). Salzburg: 13-60.

1973b «Das Schöpfungswort in der vedischen Kosmologie», in: Musicae scientiae collectanea, Festschrift für K.G. Fellerer. Köln: 523-526.

1974 «Der wahre Don Juan», Zeitschrift für Ganzheitsforschung (Wien).

1976 Le chant des pierres. Études sur le rythme et la signification des chapiteaux dans trois cloûtres catalans de style roman. Milano: Archè.

1978 Singende Steine: Rythmus-Studien an drei romanischen Kreuzgängen. München: Heimeran. 123 p. (2. erw. Aufl.).

1979 «Klangsymbolik in fremden Kulturen», Beiträge zur harmonikalen Grundlagenforschung (Wien).

1980 «La symbologia del asino», Conoscenza religiosa.

1 Certaines références nous ont été communiquées de façon incomplète et il nous a hélàs été impossible de les complèter. Nous avons néanmoins résolu de les maintenir dans cette bibliographie en raison de leur intérêt particulier (ndlr). 
1980a «Urweltmythos und Sphärenharmonie», in: Festschrift für R. Haase (Eisenstadt): 95ff.

1981 «Lobopfer, sacrificium laudis (Vom Urrhytmus zum gregorianischen Choral)», in: B. Moser (Hrsg.), Das christliche Universum (München): 14-23.

1982 "On Gregorian chant and the human voice», The world of music XXIV, 3: 3-21.

1984 "The birth of the symbol in music», The world of music XXVI, 3: 3-21.

1985 «Die Musik im symbolischen Denken», in: Organismus und Technik (Luzern), LoseblattFolge Nr. 28.

1990 «Kosmogonie», in: Jahrbuch für musikalische Volks- und Völkerkunde (Kassel) 14: 9-51. 